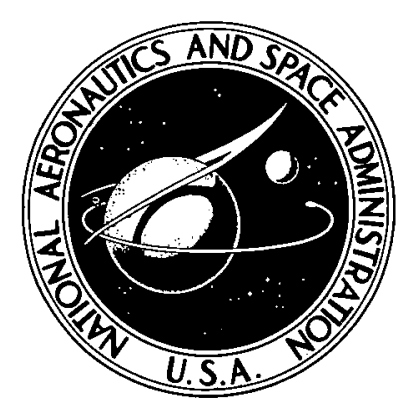

NASA İN D-7239

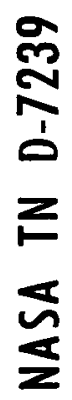

ROLE OF GAS-SURFACE INTERACTIONS

IN THE REDUCTION OF OGO 6

NEUTRAL-PARTICLE MASS SPECTROMETER DATA

by

A. E. Hedin

Goddard Space Flight Center

Greenbelt, Md. 20771

and

B. B. Hinton and G. A. Schmitt

University of Michigan

Ann Arbor, Mich. 48103

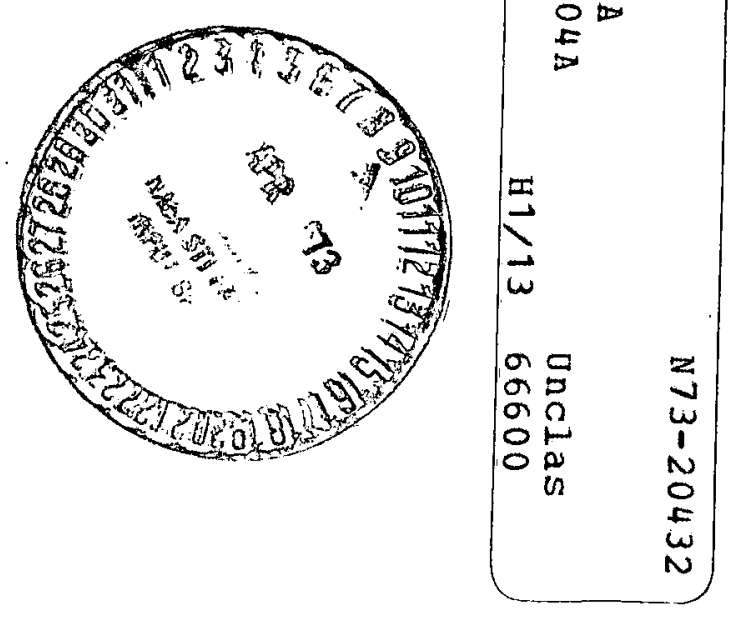

NATIONAL AERONAUTICS AND SPACE ADMINISTRATION - WASHINGTON, D. C. - MARCH 1973 


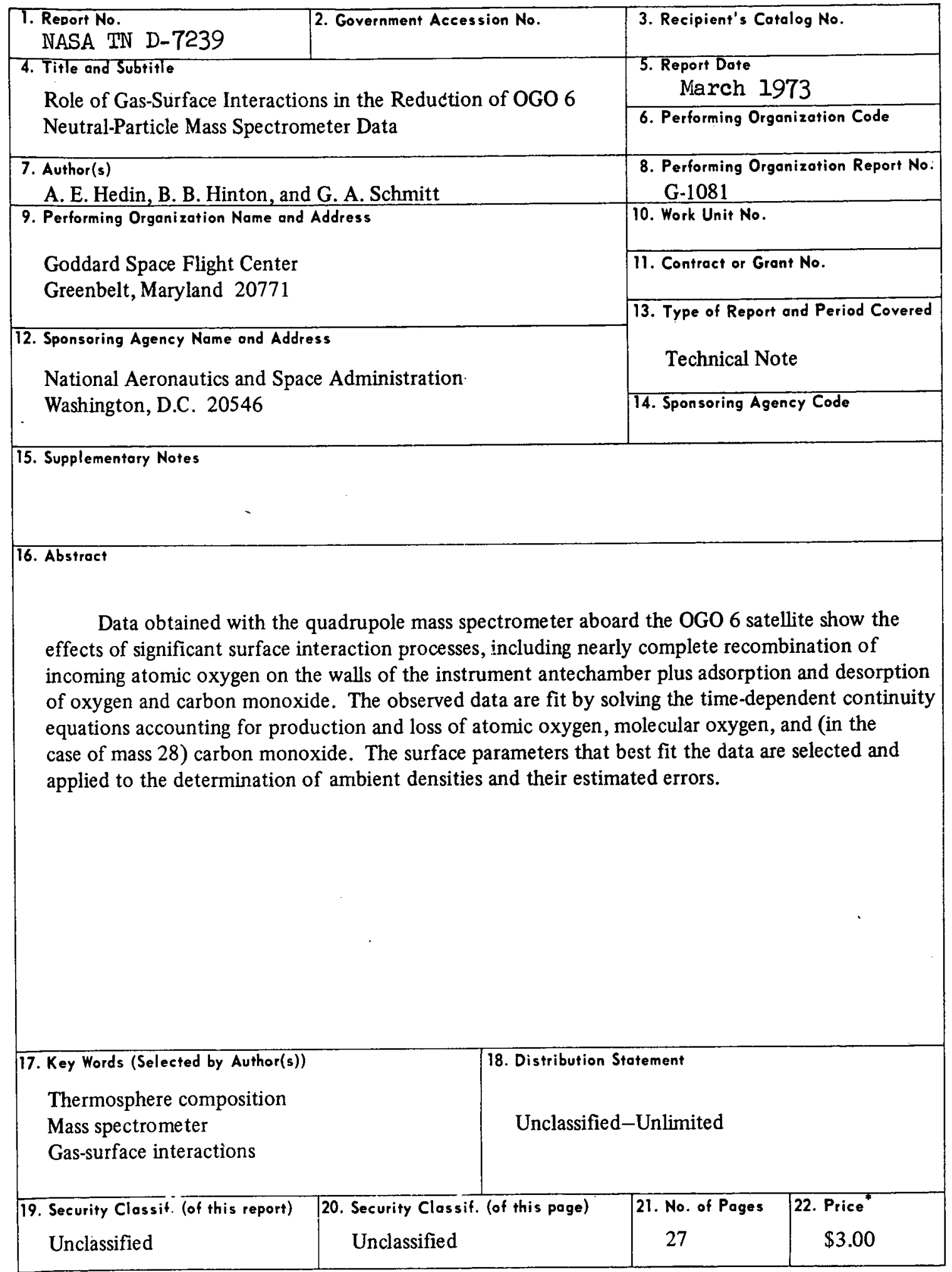

* For sale by the National Technical Information Service, Springfield, Virginia 22151. 


\section{CONTENTS}

ABSTRACT . . . . . . . . . . . . . . . . . . . . . . . . . . . . . . . . .

INTRODUCTION . . . . . . . . . . . . . . . . . . . . . . . . . . . . . . . . . . . . . . .

DESCRIPTION OF THE EXPERIMENT . . . . . . . . . . . . . . . . . . . . . . . . . . . . 1

GENERAL OBSERVATIONS . . . . . . . . . . . . . . . . . . . . . . . . . . . . . . . 2

MOLECULAR NITROGEN DENSITY . . . . . . . . . . . . . . . . . . . . . . . . . 6

ATOMIC OXYGEN DENSITY . . . . . . . . . . . . . . . . . . . . . . . . . . . . . . 15

REFERENCES . . . . . . . . . . . . . . . . . . . . . . . . . . . . . . . 27 


\title{
ROLE OF GAS-SURFACE INTERACTIONS IN THE REDUCTION OF OGO 6 NEUTRAL-PARTICLE MASS SPECTROMETER DATA
}

\author{
A. E. Hedin \\ Goddard Space Flight Center \\ B. B. Hinton and G. A. Schmitt \\ University of Michigan
}

\section{INTRODUCTION}

Several authors have pointed out that gas-surface interactions complicate the interpretation of neutral-particle density measurements in the thermosphere (Moe and Moe, 1967, 1969; Silverman and Newton, 1970; von Zahn, 1967). This paper describes the gas-surface interaction effects observed by a satellite neutral-particle mass spectrometer and summarizes the technique developed to account for them in determining ambient neutral densities.

\section{DESCRIPTION OF THE EXPERIMENT}

The Orbiting Geophysical Observatory (OGO) 6 is a polar-orbiting satellite with an inclination of $82^{\circ}$ prograde, an apogee of approximately $1100 \mathrm{~km}$, and a perigee of about $400 \mathrm{~km}$. It was launched on June 5, 1969 (day 156 of 1969), and was operated until July 1971. The neutral-particle mass spectrometer is mounted in an orbital-plane experiment package (OPEP) such that in the normal mode of operation, the spectrometer inlet faces the spacecraft's direction of travel. In this mode, the flux of ambient neutral molecules entering the spectrometer is modulated primarily by the varying ambient density as the spacecraft changes altitude and position. On command, the OPEP can be rotated cyclically through $220^{\circ}$ about a vertical axis, effecting an angle-of-attack modulation of the incoming flux of ambient molecules.

The quadrupole mass spectrometer employs an electron-impact ion source behind a gold-plated stainless-steel antechamber (fig. 1). Ambient neutral molecules enter the antechamber through a knifeedged orifice and undergo many surface collisions before entering the ion source region. Ions created by impact with a beam of $75-\mathrm{V}$ electrons in the source region are focused by an electrostatic lens and filtered according to their charge-to-mass ratios. Details of the instrument construction and operation have been documented elsewhere (Carignan and Pinkus, 1968).

In the normal mode, the total ion current and the ion currents for ions with molecular weights 2 , $4,16,28$, and 32 are sampled for 1.152 s once every 9.216 s during 258 s out of a 368 -s cycle. During $110 \mathrm{~s}$ of the 368 -s cycle the spectrometer is in sweep mode, during which period each ion in the range 


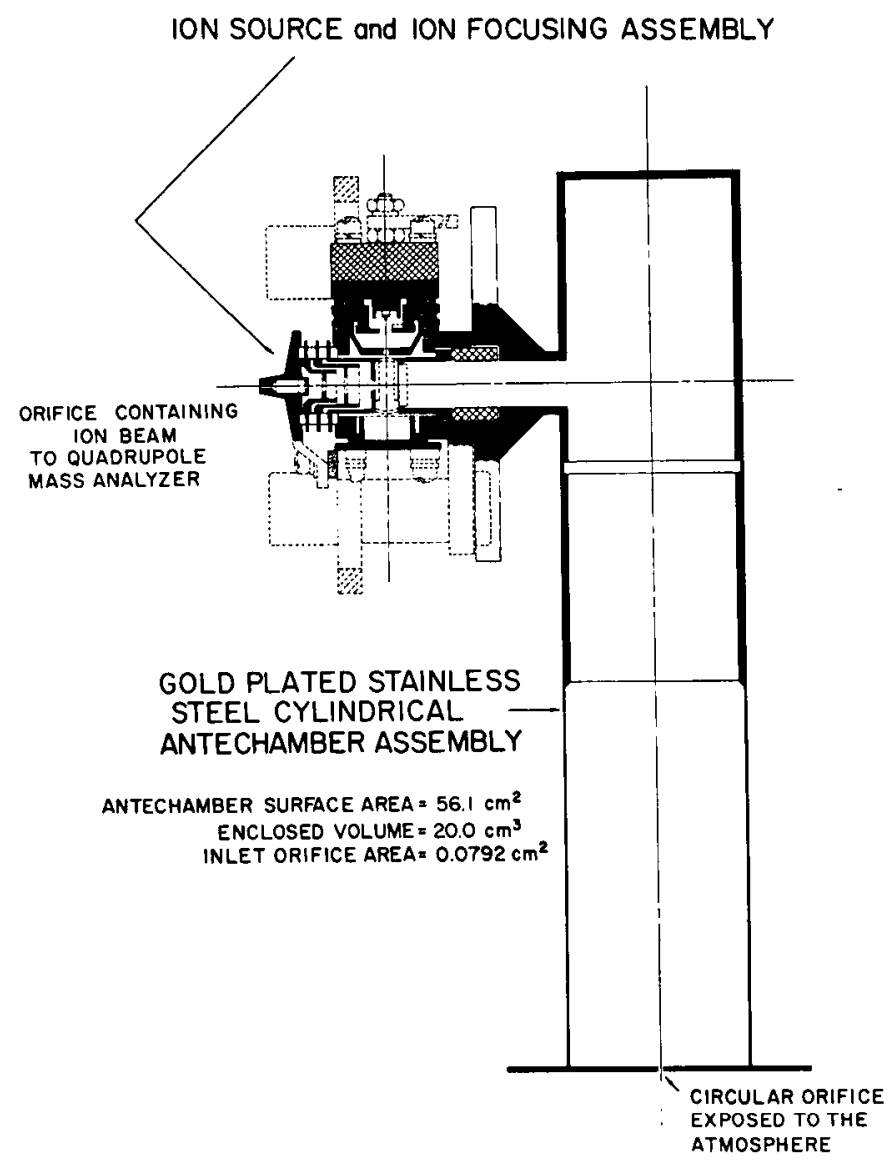

Figure 1.-Cross section of mass spectrometer antechamber and ion source.

from 1 to $46 \mathrm{amu}$ is sampled at least twice. A continuous-sweep mode is available on command, and masses of $2,4,28,32$, or the total flux can be sampled continuously on command.

\section{GENERAL OBSERVATIONS}

Based on the assumption that incoming ambient molecules are thermally accommodated to the antechamber surface temperature, the following equation gives the number density as a function of time $t$ for any constituent in the ion source region:

where

$$
\frac{d n_{g}}{d t}=\frac{(1 / 4) n_{a} F(s) \bar{c}_{a} A_{h}-(1 / 4) n_{g} \bar{c} A_{h}}{V}
$$

$$
F(s)=\frac{2 \pi^{1 / 2} v_{n}}{\bar{c}_{a}} \quad \text { for } v_{n} \gg \bar{c}_{a}
$$


$v_{n}=$ spacecraft velocity component normal to the inlet orifice

$n_{g}=$ ion source number density

$n_{a}=$ ambient number density

$A_{h}=$ area of the inlet knife-edged orifice

$\bar{c}=$ mean thermal speed of constituen't molecules at the antechamber surface temperature

$\bar{c}_{a}=$ mean thermal speed of constituent molecules in the ambient gas

$V=$ volume enclosed by the antechamber

Equation (1) describes the balance of molecular fluxes into and out of the spectrometer and may be used to consider the response of the source density to changes in incoming flux. For example, if the incoming flux decreases to zero, then

$$
\frac{d n_{g}}{d t}=-\frac{\bar{c} A_{h}}{4 V} n_{g}
$$

and the source density would decrease exponentially with a time constant of $4 V / \bar{c} A_{h}$. This value for the OGO 6 mass spectrometer is of the order of $0.02 \mathrm{~s}$. Physically, this means that the antechamber should be emptied of molecules almost immediately if the incoming flux is stopped.

A direct test of the simple model represented by equation (1) is provided by a comparison of figures 2(a) and (b). Figure 2(a) is a plot showing typical source-density data taken during one orbit of the OGO 6 spacecraft. The observed source densities plotted in figure 2(a) were obtained by multiplying the ion current for each constituent by the laboratory sensitivity for that constituent. Figure 2(b) is an equivalent plot using equation (1) together with a model atmosphere prediction of ambient densities as a function of altitude.

Figure 2(a) indicates that atomic oxygen has combined in large part to form molecular oxygen, which appears as an ion current at mass 32 and a smaller ion current at mass 16. Mass 16 values are largely the result of fractionation of molecular oxygen in the source region. Both mass 32 and mass 28 have a maximum source density near perigee as expected from the strong dependence of neutral ambient density on altitude. There is, however, an asymmetry in the source density about perigee due to surface absorption processes before perigee and desorption processes after perigee. All the constituents except helium have detectable source densities near apogee as a result of desorption. Finally, the presence of mass $44\left(\mathrm{CO}_{2}\right.$ or $\left.\mathrm{N}_{2} \mathrm{O}\right)$ and mass 1 (atomic hydrogen), as well as carbon monoxide, is thought to result from chemical reactions of atmospheric atomic oxygen with constituents on the instrument surfaces.

Figure 3 shows some long-term features of the measured source-density data. The perigee and apogee source densities during an orbit are plotted versus time for ions with masses 16, 28, and 32 . The perigee density data reflect the changing atmosphere as the spacecraft perigee changes latitude and local time, and as the atmosphere itself varies. The apogee source density is higher than simple theory predicts (fig. 2(b)) and depends to some extent on the magnitude of perigee density. It was also found 


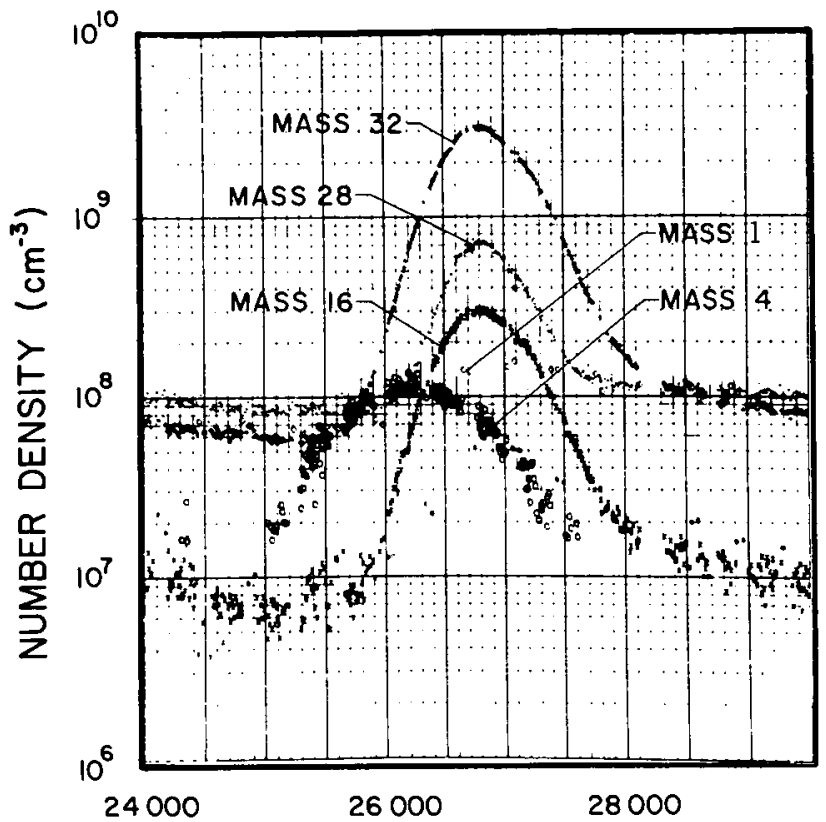

(a)
TIME (s)

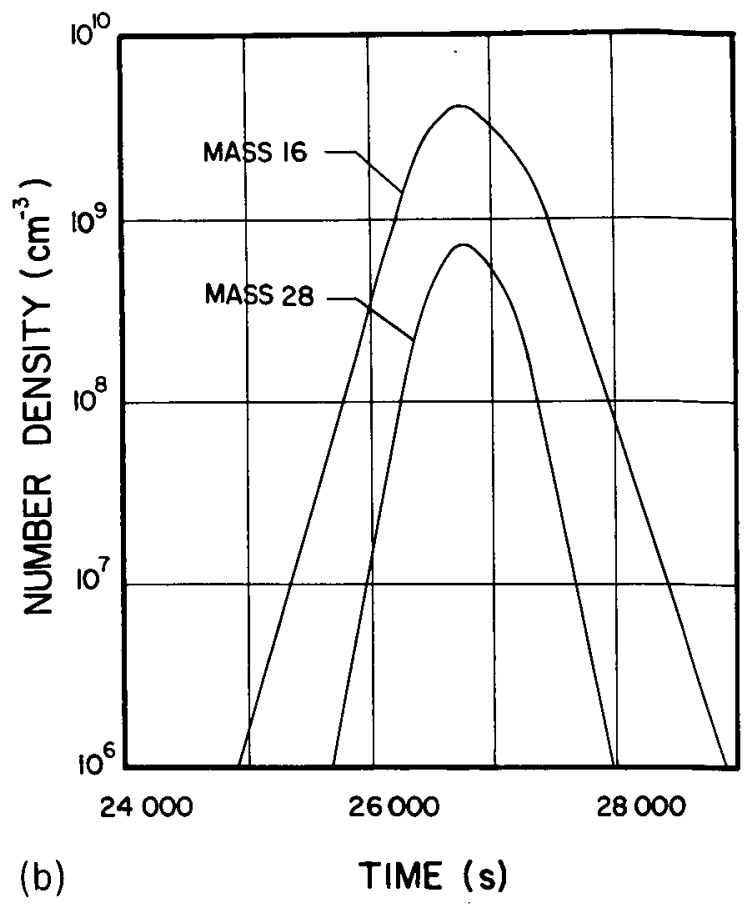

(b)

TIME (s)

Figure 2.-Source densities during orbit. (a) Observed, for typical orbit. (b) Hypothetical, without gas-surface interactions.

that, after an initial period, the maximum densities for masses 16 and 32 have a nearly constant ratio of 0.1 , indicating a common source for these two constituents and a certain stability in the processes leading to these signals. Also, the long-term trend in minimum density for mass 28 is decreasing, unlike mass 32 , indicating that the processes determining minimum densities are not the same for these two components.

Several problems arose during the operation of the experiment that affect understanding of the gas-surface interactions in the spectrometer. At approximately $30 \mathrm{~s}$ after turn-on there was a sudden loss of sensitivity by approximately a factor of 5 (5.03 for mass $32,4.70$ for mass $28,1.82$ for mass $16,4.32$ for mass 4 , and 3.46 for mass 2 ). The exact cause was never determined but was believed to have originated in arcing nearby or within the experiment electronics. No indication of a further change in sensitivity was found and all densities have been adjusted for this sensitivity change. The change in sensitivity was nearly the same for every mass except 16 , and the mass 16 to mass 32 sourcedensity ratio before the shift was near the value expected from fractionation (0.0455). Thus it must be assumed that the fractionation ratio of molecular oxygen increased at this instant to 0.16 . Later, however, the 16 to 32 ratio was always lower than this value. Despite various laboratory tests on a prototype instrument, this failure pattern could not be reproduced and, therefore, the detailed mechanism is not clear. The observation of a constant 16 to 32 ratio after the first 2 weeks indicates that the new sensitivities have been stable. Nevertheless, the difficulty in determining the correct value to be used for the fractionation pattern of oxygen contributes to the inability to uniquely determine certain surface parameters. 


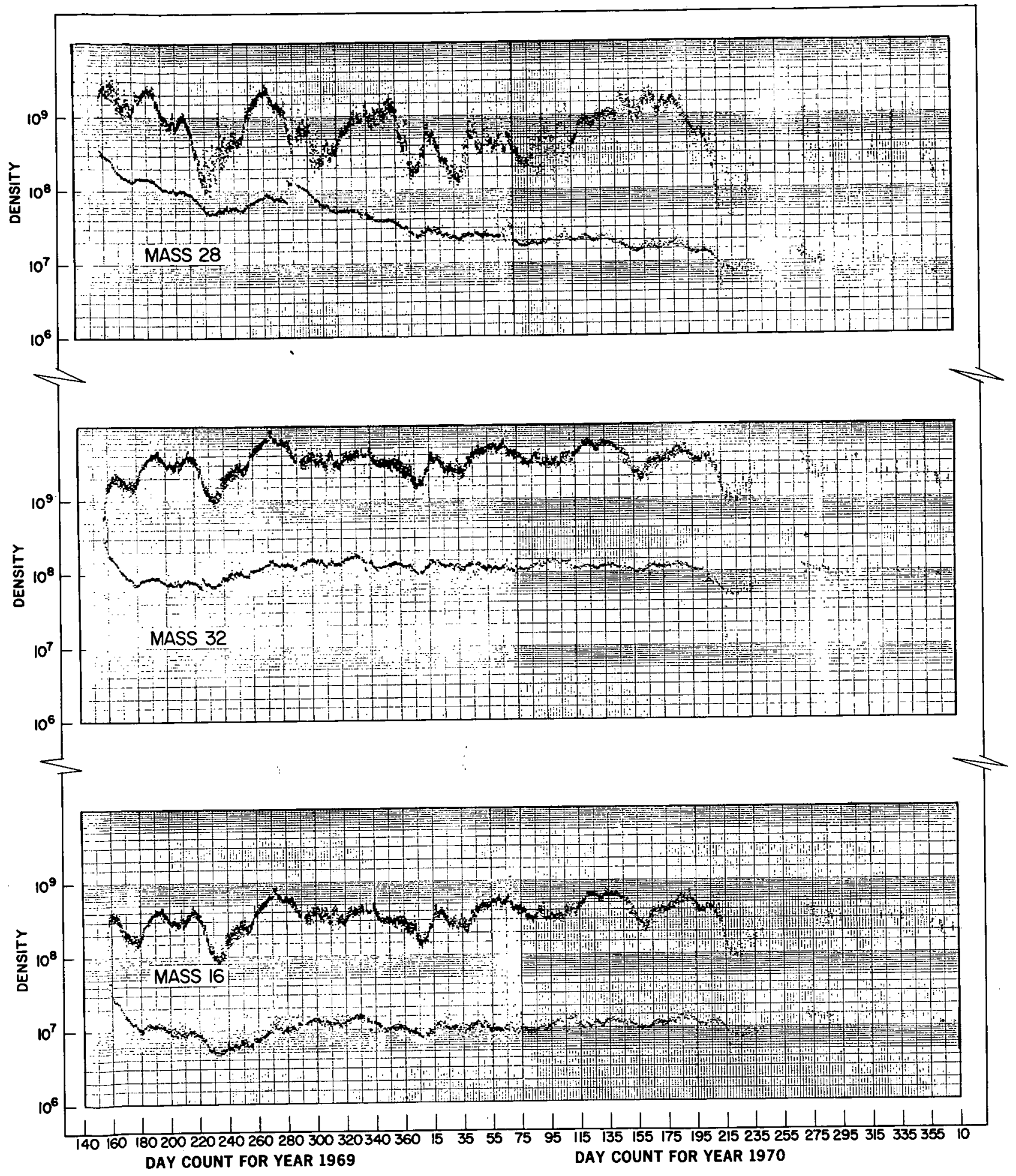

Figure 3.-Perigee and apogee source densities for constituent masses 28, 32, and 16. 
On day 225 of 1969 , an electronic failure occurred which put the quadrupole spectrometer into an anomalous operating mode during the measurement of mass 32 . The net result was that the ion current recorded for mass 32 became the total ion current for masses greater than 27 . To compensate the data acquired after this failure, the mass 28 ion current was subtracted from the mass 32 ion current in the analysis process. Unfortunately, there were some small currents at other masses, particularly 30 and 44, which could not be determined with the frequency or accuracy needed to permit subtraction from the measured mass 32 ion current. The mass 32 source density measurement thus contained a residual contribution from these other masses, as seen by the jump in apogee value at day 225 in figure 3. The principal effect on ambient density determination is not from the direct contribution, which is negligible near perigee, but in the error it may cause in surface parameter determination.

The temperature of the antechamber is of interest because the surface desorption rate is an exponential function of temperature (Ehrlich, 1959). A thermistor, located near the source region (fig. 1), recorded changes in temperature of $\pm 10 \mathrm{~K}$ with a nominal value of $293 \mathrm{~K}$. These temperature variations are sufficiently large to produce noticeable changes in desorption-nearly a factor of 4 for a desorption energy of $92 \mathrm{~kJ}$ ( $22 \mathrm{kcal})$ per mole. However, the measured temperature may not accurately reflect the effective antechamber temperature because, for example, the measured temperature was minimized when the angle between the sun and the front of the antechamber was smallestopposite to the anticipated behavior. The measured temperature was ignored in the final surface analysis, but these data were examined for possible examples of thermal effects.

\section{MOLECULAR NITROGEN DENSITY}

Analysis of gas-surface interactions affecting the mass 28 source density depends critically upon whether the ion current at mass 28 is due primarily to $\mathrm{N}_{2}$ or $\mathrm{CO}$. If the mass 28 ion current is entirely due to $\mathrm{N}_{2}$, then one could use an adsorption model for $\mathrm{N}_{2}$ of the type discussed by Moe and Moe $(1967,1969)$ in connection with the analysis of pressure-gage data. The net result will be an additive correction before perigee and a subtractive correction after perigee. If, on the other hand, the mass 28 ion current at apogee is due primarily to $\mathrm{CO}$, one must consider the adsorption-desorption reactions of this gas rather than $\mathrm{N}_{2}$, and the correction will always be subtractive.

The most direct determination of apogee mass 28 peak composition would come, in principle, from comparing the mass 14 and 28 ion currents. Mass 14 ion current results from dissociative ionization of $\mathrm{N}_{2}$, while mass 28 ion current results from both $\mathrm{N}_{2}$ and $\mathrm{CO}$. Early in the spacecraft's lifetime, the ion current measured at mass 14 indicated that $\mathrm{CO}$ was present near perigee. During this early period there were substantial ion currents at mass 44 , indicating the probable presence of neutral $\mathrm{CO}_{2}$. Unfortunately, at high altitudes where the concentration of $\mathrm{N}_{2}$ in the atmosphere is negligible, the ion current at mass 14 was too small to identify the neutral constituent contributing to the mass 28 ion current. The drop in sensitivity referred to earlier resulted in a generally undetectable mass 14 ion current at apogee after the first few days. If the apogee mass 28 ion current were entirely due to $\mathrm{N}_{2}$, the expected mass 14 ion current would have produced only one or two bits of telemetry signal.

However, the identification of the mass 28 background gas as $\mathrm{CO}$ is supported by other evidence. Laboratory experience indicates that $\mathrm{CO}$ is almost always present to some degree in evacuated systems. The long-term downward trend in the apogee mass 28 source density is not compatible with the apogee 
density resulting from $\mathrm{N}_{2}$ desorption, unless an ad hoc change in the desorption rate coefficient is postulated. Furthermore, there was a definite, measurable increase in $\mathrm{CO}$ at filament change on day 286 of 1969 , thus indicating the continuing importance of CO. Finally, previous laboratory work on surface interactions (Trapnell, 1953) indicates that gold is the least reactive of all metals and $\mathrm{N}_{2}$ the least reactive molecule (except for the noble gases) on any metal, whereas $\mathrm{CO}$ is known to chemisorb on gold.

To develop a definite model, one may suppose that there exists an initial surface density of carbon that is slowly replaced by diffusion as it is depleted by reaction with oxygen atoms. The base metal, stainless steel, would be a reservoir for carbon that could slowly diffuse through the base metal and the gold-over-nickel surface layer. Extrapolation of available data indicates that the total CO generated in the instrument will consume a number of carbon atoms equivalent to at least a few parts per million of the antechamber base metal.

It is assumed that $\mathrm{CO}$ is produced by two reactions involving wall $w$ and gas $g$ atoms:

$$
\begin{aligned}
& \mathrm{C}(w)+\mathrm{O}(g) \rightarrow \mathrm{CO}(g) \\
& \mathrm{C}(w)+\mathrm{O}(w) \rightarrow \mathrm{CO}(w)
\end{aligned}
$$

Further, it is assumed that $\mathrm{CO}$ is destroyed by three reactions:

$$
\begin{aligned}
\mathrm{CO}(w) & +\mathrm{O}(g) \rightarrow \mathrm{CO}_{2}(g) \\
\mathrm{CO}(g)+\mathrm{O}(w) & \rightarrow \mathrm{CO}_{2}(g) \\
\mathrm{CO}(w)+\mathrm{O}(w) & \rightarrow \mathrm{CO}_{2}(g)
\end{aligned}
$$

Although these reactions represent negligible sinks for oxygen atoms (after the first 20 days of flight), they do constitute a significant loss of $\mathrm{CO}$.

In writing these equations, it is assumed that some of the incoming oxygen atoms are adsorbed on the walls. Molecules (or atoms) on the wall are distinguished as a separate species (e.g., $\mathrm{CO}(w)$ ) from the ordinary, or gas phase molecules or atoms (e.g., $\mathrm{CO}(g))$. It has been assumed that there are at most two adsorbed states of $\mathrm{CO}$ and a single adsorbed state of atomic oxygen. It is thought that most chemisorption systems have multiple binding states, each with a distinct sticking coefficient (or probability) and desorption time constant. It is conceivable, for example, that the $\mathrm{CO}$ molecules adsorbed from the gas phase are bound to the surface mainly in one state and the molecules produced by adatom-adatom combination in a preadsorbed state might be bound primarily in a second state. Any attempt to account for this and for the rates of interconversion among the states increases enormously the complexity of the problem. These considerations would be appropriate for laboratory investigations where, in principle at least, conditions may be controlled and are repeatable. As far as possible, all but the dominant states have been ignored because of the lack of sufficiently detailed information to consider more than one or two surface states.

The following expressions were obtained by considering production, loss, outflow, adsorption, and desorption. In these equations and in those in the following sections, $k$ is used to denote sticking coefficients, $\sigma_{a g}$ to represent cross sections for reactions of an adsorbed constituent with one in the 
gas phase, $\sigma_{a a}$ to represent a cross section per unit time that describes the rate of reaction of two adsorbed constituents, and $\tau_{w}$ to represent the lifetime of an adsorbed constituent. A subscript $w$ on a density refers to the number per unit area on the wall; a subscript $g$ refers to an ordinary volume number density. All of these quantities are associated with a specific process by means of additional subscripts and superscripts, as indicated in the following list:

$A_{h}=$ inlet aperture area, $\mathrm{cm}^{2}$

$A_{w}=$ wall area of antechamber, $\mathrm{cm}^{2}$

$\bar{c}_{a}^{16}=$ mean thermal speed of ambient atomic oxygen, $\mathrm{cm} \mathrm{s}^{-1}$

$\bar{c}_{g}^{16}=$ mean thermal speed of atomic oxygen, $\mathrm{cm} \mathrm{s}^{-1}$

$\bar{c}_{g}^{28}=$ mean thermal speed of molecular nitrogen or carbon monoxide, $\mathrm{cm} \mathrm{s}^{-1}$

$\bar{c}_{g}^{32}=$ mean thermal speed of molecular oxygen, $\mathrm{cm} \mathrm{s}^{-1}$

$k_{16}=$ sticking probability of atomic oxygen

$k_{\mathrm{CO}}=$ sticking probability of carbon monoxide

$n_{a}^{16}=$ density of ambient atomic oxygen multiplied by the function $F(s), \mathrm{cm}^{-3}$

$n_{g}^{16}=$ density of atomic oxygen in antechamber, $\mathrm{cm}^{-3}$

$n_{g}^{32}=$ density of molecular oxygen in antechamber, $\mathrm{cm}^{-3}$

$n_{g}^{\mathrm{CO}}=$ density of carbon monoxide in antechamber, $\mathrm{cm}^{-3}$

$n_{w}^{12}=$ density of carbon atoms on walls, $\mathrm{cm}^{-2}$

$n_{w}^{16}=$ density of atomic oxygen on walls, $\mathrm{cm}^{-2}$

$n_{w}^{\mathrm{CO}}=$ density of carbon monoxide on walls, $\mathrm{cm}^{-2}$

$V=$ antechamber volume, $\mathrm{cm}^{3}$

$\sigma_{a a}^{32}=$ adatom-adatom recombination coefficient, $\mathrm{cm}^{2} \mathrm{~s}^{-1}$

$\sigma_{a g}^{32}=$ adatom-gas atom recombination coefficient, $\mathrm{cm}^{2}$

$\sigma_{a a}^{\mathrm{CO}}=$ coefficient of $\mathrm{CO}$ production by $\mathrm{C}(w)+\mathrm{O}(w), \mathrm{cm}^{2} \mathrm{~s}^{-1}$

$\sigma_{a g}^{\mathrm{CO}}=$ coefficient of $\mathrm{CO}$ production by $\mathrm{C}(w)+\mathrm{O}(\mathrm{g}), \mathrm{cm}^{2}$

$\sigma_{a a}^{\mathrm{CO}_{2}}=$ coefficient of $\mathrm{CO}_{2}$ production by $\mathrm{CO}(w)+\mathrm{O}(w), \mathrm{cm}^{2} \mathrm{~s}^{-1}$

$\sigma_{a g}^{\mathrm{CO}_{2}}=$ coefficient of $\mathrm{CO}_{2}$ production by $\mathrm{CO}(w)+\mathrm{O}(\mathrm{g}), \mathrm{cm}^{2}$

$\sigma_{g a}^{\mathrm{CO}_{2}}=$ coefficient of $\mathrm{CO}_{2}$ production by $\mathrm{CO}(g)+\mathrm{O}(w), \mathrm{cm}^{2}$

$1 / \tau_{w}^{16}=$ desorption rate of atomic oxygen, $\mathrm{s}^{-1}$

$1 / \tau_{w}^{\mathrm{CO}}=$ desorption rate of carbon monoxide, $\mathrm{s}^{-1}$ 
Outflow is sufficiently rapid that the steady-state assumption applies to $n_{g}^{\mathrm{CO}}$. Consequently

$$
\begin{aligned}
& \frac{d n_{g}^{\mathrm{CO}}}{d t}=\frac{n_{g}^{16} \bar{c}_{g}^{16} n_{w}^{12} A_{w}}{4 V} \sigma_{a g}^{\mathrm{CO}}+\frac{n_{w}^{\mathrm{CO}} A_{w}}{V \tau_{w}^{\mathrm{CO}}}-\frac{n_{g}^{\mathrm{CO}} \bar{c}_{g}^{28}}{4 V}\left(k_{\mathrm{CO}} A_{w}+A_{h}+\sigma_{g a}^{\mathrm{CO}} n_{w}^{16} A_{w}\right)=0 \\
& \frac{d n_{w}^{\mathrm{CO}}}{d t}=n_{w}^{12} \sigma_{a a}^{\mathrm{CO}} n_{w}^{16}+\frac{k_{\mathrm{CO}} n_{g}^{\mathrm{CO}} \bar{c}_{g}^{28}}{4}-n_{w}^{\mathrm{CO}}\left(\frac{1}{\tau_{w}^{\mathrm{CO}}}+\sigma_{a a}^{\mathrm{CO}} n_{w}^{16}+\frac{\sigma_{a g}^{\mathrm{CO}_{2}} \bar{c}_{g}^{16} n_{g}^{16}}{4}\right)
\end{aligned}
$$

Rearrangement of equation (4) results in

$$
n_{g}^{\mathrm{CO}}=\frac{4 n_{w}^{\mathrm{CO}} / \tau_{w}^{\mathrm{CO}}+\sigma_{a g}^{\mathrm{CO}} n_{g}^{16} \bar{c}_{g}^{16} n_{w}^{12}}{\bar{c}_{g}^{28}\left(k_{\mathrm{CO}} A_{w}+A_{h}+\sigma_{g a}^{\mathrm{CO}} 2 n_{w}^{16} A_{w}\right)} A_{w}
$$

if $d n_{g}^{\mathrm{CO}} / d t$ is negligible in comparison to the other terms in the equation. If one considers $n_{g}^{16}$ and $n_{w}^{16}$ to be explicitly known functions of time, equation (5) can be solved for $n_{w}^{\mathrm{CO}}$ if $n_{g}^{\mathrm{CO}}$ is replaced by equation (6). If one lets

$$
L(t)=\frac{1}{\tau_{w}^{\mathrm{CO}}}+\sigma_{a a}^{\mathrm{CO}} n_{w}^{16}+\frac{1}{4} \sigma_{a g}^{\mathrm{CO}_{2}} \bar{c}_{g}^{16} n_{g}^{16}-\frac{k_{\mathrm{CO}} A_{w}}{\tau_{w}^{\mathrm{CO}}\left(k_{\mathrm{CO}} A_{w}+A_{h}+\sigma_{g a}^{\left.\mathrm{CO}_{2} n_{w}^{16} A_{w}\right)}\right.}
$$

and

$$
P(t)=n_{w}^{12}\left(\sigma_{a a}^{\mathrm{Co}} n_{w}^{16}+\frac{k_{\mathrm{CO}}}{4} \frac{n_{g}^{16} \bar{c}_{g}^{16} A_{w} \sigma_{a g}^{\mathrm{CO}}}{k_{\mathrm{CO}} A_{w}+A_{h}+\sigma_{g a}^{\mathrm{CO}} n_{w}^{16} A_{w}}\right)
$$

then

$$
\frac{d n_{w}^{\mathrm{CO}}}{d t}+n_{w}^{\mathrm{CO}} L(t)=P(t)
$$

and the solution is

$n_{w}^{\mathrm{CO}}=\exp \left[-\int_{-\infty}^{t} L\left(t^{\prime}\right) d t^{\prime}\right] \int_{-\infty}^{t} P\left(t^{\prime}\right) \exp \left[\int_{-\infty}^{t^{\prime}} L\left(t^{\prime \prime}\right) d t^{\prime \prime}\right] d t^{\prime}+c \exp \left[-\int_{-\infty}^{t} L\left(t^{\prime}\right) d t^{\prime}\right]$

where $c$ is a parameter, discussed later. After sufficient time

$$
n_{w}^{\mathrm{CO}}=\exp \left[-\int_{-\infty}^{t} L\left(t^{\prime}\right) d t^{\prime}\right] \cdot \int_{-\infty}^{t} P\left(t^{\prime}\right) \exp \left[\int_{-\infty}^{t^{\prime}} L\left(t^{\prime \prime}\right) d t^{\prime \prime}\right] d t^{\prime}
$$

Use of this result with equation (6) yields

$$
n_{g}^{\mathrm{CO}}=\frac{\sigma_{a g}^{\mathrm{CO}} n_{g}^{16} \bar{c}_{g}^{16} n_{w}^{12} A_{w}+\left(4 A_{w} / \tau_{w}^{\mathrm{CO}}\right)\left\{P * \exp \left[\int_{-\infty}^{t} L\left(t^{\prime}\right) d t^{\prime}\right]\right\}}{\tau_{w}^{\mathrm{CO}} \bar{c}_{g}^{28}\left(k_{\mathrm{CO}} A_{w}+A_{h}+\sigma_{g a}^{\mathrm{CO}} n_{w}^{16} A_{w}\right)}
$$

in which $*$ denotes convolution. 
In the investigation of $\mathrm{CO}$, using a heuristic approach, it was discovered that the $\mathrm{CO}$ density could be approximated by an expression of the form

$$
n_{g}^{\mathrm{CO}} \approx a^{\prime} n_{g}^{32}+\frac{b^{\prime}}{\tau} \int_{-\infty}^{t} n_{g}^{32} \exp \left(\frac{t^{\prime}-t}{\tau}\right) d t^{\prime}+c
$$

The $\mathrm{CO}$ density during an orbit was determined by subtracting the mass 14 source density from the mass 28 source density. The mass 14 source density took into account the fractionation ratio and should have agreed with the mass 28 source density if no CO were present. Also, the mass 28 source density within $1500 \mathrm{~s}$ of apogee was taken to indicate $\mathrm{CO}$ density, consistent with the earlier decision on the composition of the apogee mass 28 density. After several months, the mass 14 source density near perigee was consistently higher than the mass 28 source density by about 11.4 percent. There was no way to determine from the available measurements whether this was due to an error in the fractionation ratio or to a true, ambient, atomic nitrogen density. It was decided to adjust the fractionation ratio to make the two source densities agree during the later orbits.

Equation (13) can be interpreted in terms of a simplified solution of equation (12) in which $\sigma_{a a}^{\mathrm{CO}}, \sigma_{a a}^{\mathrm{CO}_{2}}, \sigma_{a g}^{\mathrm{CO}_{2}}$, and $\sigma_{g a}^{\mathrm{CO}_{2}}$ are ignored and $n_{g}^{32}$ is assumed to be a rough measure of $n_{g}^{16}$. The resulting solution for $n_{g}^{\mathrm{CO}}$ is given by equation (14), in which $k_{\mathrm{CO}}^{\prime}=k_{\mathrm{CO}} A_{w} / A_{h}$, a modified sticking coefficient, $\tau_{w}^{\mathrm{CO}^{\prime}} \stackrel{g}{=}\left(1+k_{\mathrm{CO}}^{\prime}\right) \tau_{w}^{\mathrm{CO}}$, a modified desorption time constant, and $a=a^{\prime}\left(1+k_{\mathrm{CO}}^{\prime}\right)$, a parameter proportional to $n_{w}^{12}$ :

$$
n_{g}^{\mathrm{CO}} \approx a\left[\frac{n_{g}^{32}}{1+k_{\mathrm{CO}}^{\prime}}+\frac{k_{\mathrm{CO}}^{\prime}}{1+k_{\mathrm{CO}}^{\prime}} \frac{1}{\tau_{w}^{\mathrm{CO}}} \int_{-\infty}^{t} n_{g}^{32} \exp \left(\frac{t^{\prime}-t}{\tau_{w}^{\mathrm{CO}^{\prime}}}\right) d t^{\prime}\right]+c
$$

Only during the first 2 weeks of flight were there sufficient $\mathrm{CO}$ data near perigee to allow a determination of all four unknown parameters. The data were grouped into half-day intervals, and parameters $a, k_{\mathrm{CO}}^{\prime}$, and $c$ were determined for various fixed values of $\tau_{w}^{\mathrm{CO}^{\prime}}$. A value of $1500 \mathrm{~s}$ for $\tau_{w}^{\mathrm{CO}^{\prime}}$ provided the best fit to the data. An example of the type of fit obtained is shown in figure 4,

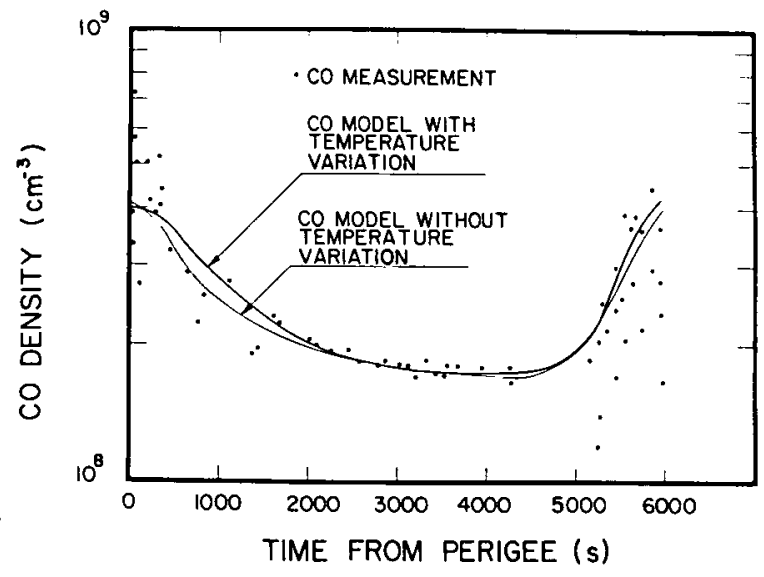

Figure 4.-Measured CO during a typical early orbit compared with model estimates. 


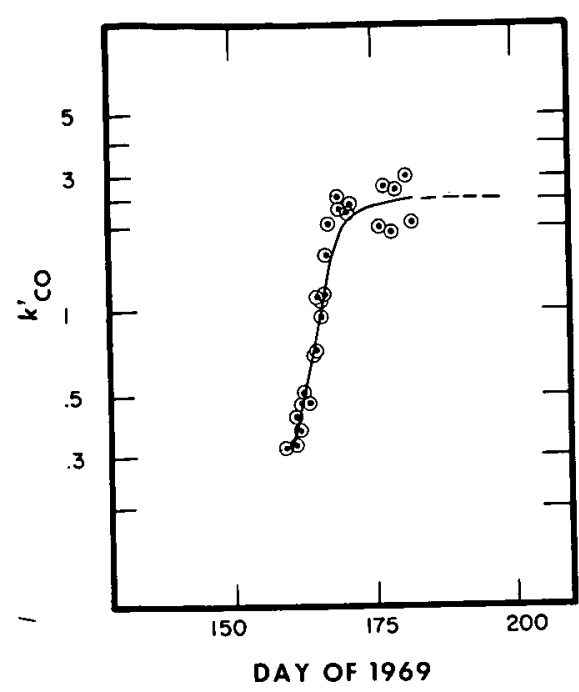

Figure 5.-Variation of parameter $k_{\mathrm{CO}}^{\prime}$ for the period when CO density was available near perigee.

including the effect of measured antechamber temperatures. Because temperature variations over the early orbits were small, it made little difference whether they were included or not. After launch on day 156 , the value of $k_{\mathrm{CO}}^{\prime}$ varied daily as shown in figure 5. It appeared to level off to a value of about 2.5 by day 180 and was impossible to determine after that. After day 180 , both $\tau_{w}^{\mathrm{CO}^{\prime}}$ and $k_{\mathrm{CO}}^{\prime}$ were held fixed; parameters $a$ and $c$ were allowed to vary, and values were then determined from all the data in a day. Thus, after day 180 it was assumed that

$$
n_{g}^{\mathrm{CO}} \approx a\left[\frac{n_{g}^{32}}{3.5}+\frac{2.5}{3.5} \frac{1}{1500} \int_{-\infty}^{t} n_{g}^{32} \exp \left(\frac{t^{\prime}-t}{1500}\right) d t^{\prime}\right]+c
$$

It is probable that the parameter $c$ in equations (13) through (15) represents a process with a time constant long compared to the orbital period. That is, $c$ is not a true constant, but might represent a quantity that changes very little around an orbit. This leads one to suppose that two surface states might be significant for $\mathrm{CO}$, with $c$ representing the more slowly changing one and the expression

$$
\frac{b^{\prime}}{\tau} \int_{-\infty}^{t} n_{g}^{32} \exp \left(\frac{t^{\prime}-t}{\tau}\right) d t^{\prime}
$$

the faster one. If this is the case, each surface state must be considered as a separate species governed by its own set of equations having the form of equations (5), (9), (10), and (11). After the appropriate modifications to equations (4) and (6), the solution for $n_{g}^{\mathrm{CO}}$ of equation (12) is replaced by

$$
n_{g}^{\mathrm{CO}}=\frac{\bar{c}_{g}^{16} n_{g}^{16} n_{w}^{12} A_{h} \sigma_{a g}^{\mathrm{CO}}+4 A_{w}\left\{P * \exp \left[-\int_{-\infty}^{\tau} L\left(t^{\prime}\right) d t^{\prime}\right] / \tau_{w}^{\mathrm{CO}}+P^{\prime} * \exp \left[-\int_{-\infty}^{t} L^{\prime}\left(t^{\prime}\right) d t^{\prime}\right] / \tau_{w}^{\mathrm{CO}}\right\}}{\left(k_{\mathrm{CO}}+k_{\mathrm{CO}}^{\prime}\right) A_{w}+A_{h}+\sigma_{a g}^{\mathrm{CO}_{2} n_{w}^{16} A_{w}}}
$$


The symbols $P^{\prime}$ and $L^{\prime}$ refer to the second state and are defined by relations analogous to equations (7) and (8).

Equation (15) may be regarded as an approximate expansion of equation (16) in terms of the convenient function $n_{g}^{32}$. To the extent that the shape of $n_{g}^{16}$ resembles $n_{g}^{32}$, one may identify the first term of equation (15) with the first term of equation (16), the second term with the second, and so on.

The long-term variation of parameter $a$ (proportional to the surface wall density of carbon atoms) can be predicted under the assumption that this density is diffusion controlled. If $n_{w}^{12}$ arose from a diffusion of carbon atoms through the wall of the antechamber, then one would expect that $n_{w}^{12}$ would vary as $\exp \left(-\pi^{2} D t / d^{2}\right)$, where $D$ is the diffusion coefficient and $d$ is the wall thickness. The diffusion coefficient is not known precisely, but is of the order of $3 \times 10^{-10} \mathrm{~cm}^{2} \mathrm{~s}^{-1}$ at $300 \mathrm{~K}$ (Kittel, 1968).

Figure 6 is a plot of the $a$ values for the first 6 months of spacecraft operation. The curves marked A represent empirical fits to the data points of the form

$$
a=a_{1} \exp \left(\frac{-\mathrm{Dy}}{T_{1}}\right)+a_{2} \exp \left(\frac{-\mathrm{Dy}}{T_{2}}\right)
$$

where $a_{1}, a_{2}, T_{1}$, and $T_{2}$ are constants and Dy is the day number. Because of the obvious perturbations occurring at the filament change on day 286 , a second curve was used beginning on that day. The $\mathrm{B}$ curve has been calculated for comparison, using a diffusion coefficient of $3 \times 10^{-10} \mathrm{~cm}^{2} \mathrm{~s}^{-1}$, accounting approximately for the various wall thicknesses of chamber parts.

The most obvious feature of the plot shown in the figure is the cyclic variation of parameter $a$. This is not consistent with the conception that $a$ is proportional to the carbon available for reaction with the incoming oxygen. One expects this parameter to decrease monotonically with time and its profile to have a greater slope when ambient oxygen is highest. Thus, either there must be some explanation for a carbon variation of this form, or the apogee slope variation must have a contribution from some source not included in the model. The large excursions of $a$ also reflect data-analysis problems, because $a$ was determined primarily from apogee data with a low signal-to-noise ratio. Also, the

Figure 6.-Variation of parameter a found from apogee data. Curves $A$ are empirical fits to data and reflect the ion source filament change. Curve $B$ results when carbon surface-density is limited by diffusion through the thin gold film.

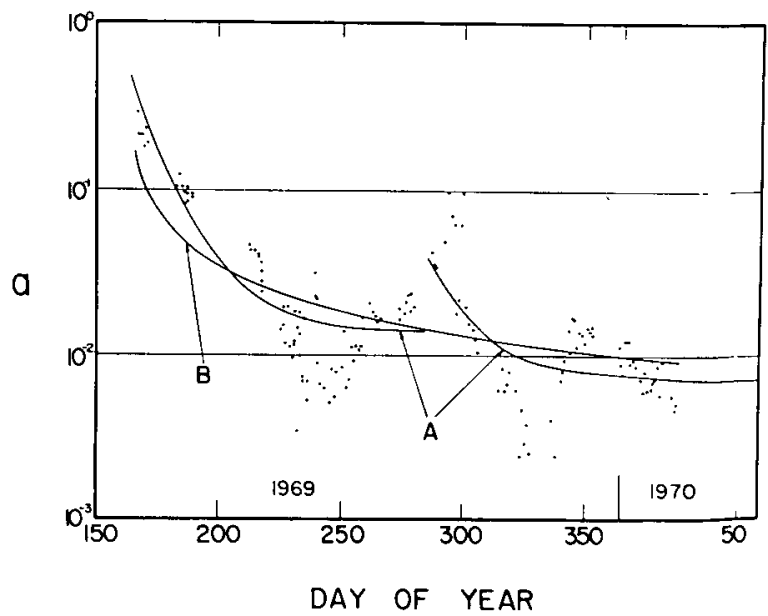




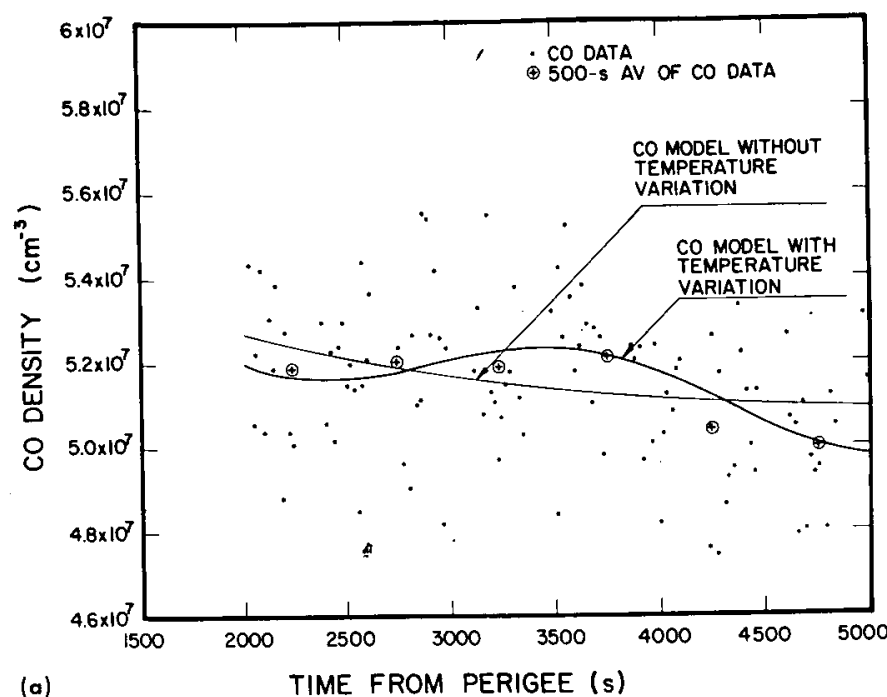

(a)

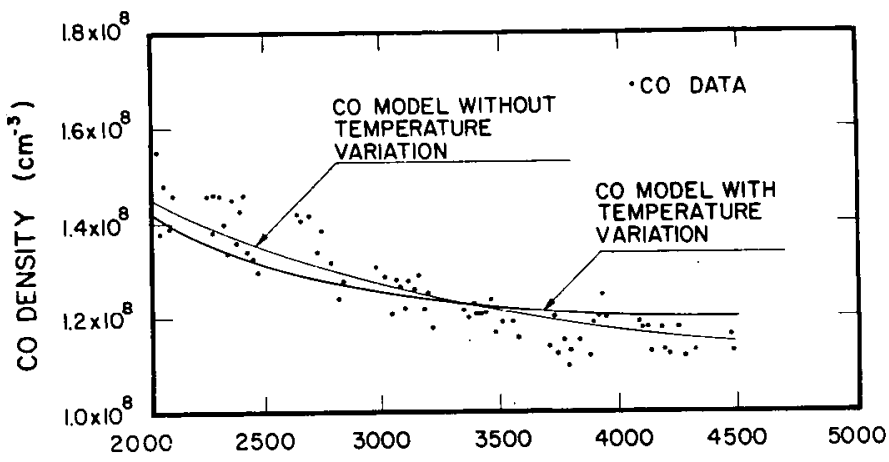

(b)

TIME FROM PERIGEE (s)

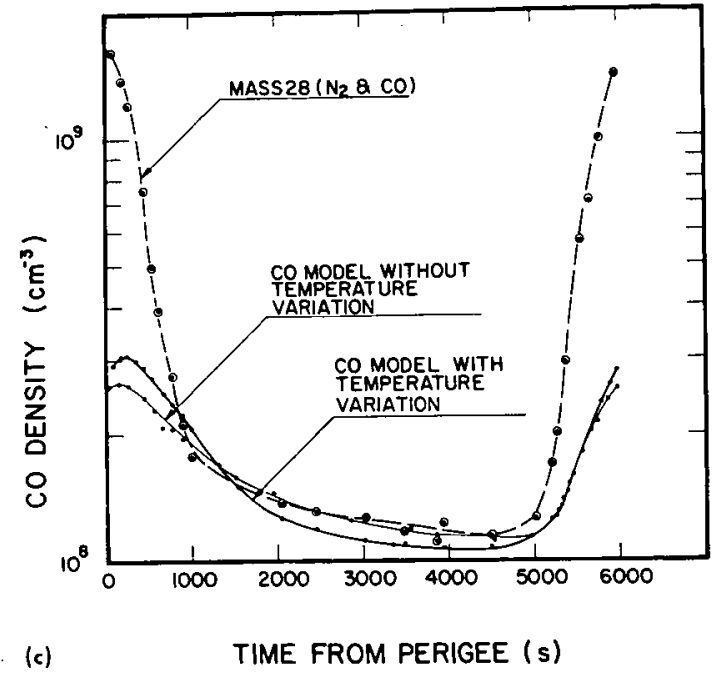

Figure 7.-Mass 28 and $\mathrm{CO}$ source densities, showing temperature effect. (a) Data near apogee. (b) Near-apogee data for different orbit. (c) Data for an entire orbit.

excursions might be artifacts of the approximations leading to equation (16). And, finally, the phenomena in question are extremely temperature dependent.

The effect of variations in surface desorption rate resulting from variations in surface temperature was studied by recalculating the $a$ parameter, using temperature as measured by the source thermistor. There was no basic change in the long-term variation of $a$. Also, individual orbits were examined to clarify the effect of including temperature. A plot of mass 28 source density near apogee is shown in figure 7 (a) compared with the best-fit prediction determined both with and without the temperature variation of desorption. Note that the sign of $d n_{g} / d t$ can be reversed by including temperature variations. While the data have considerable scatter, the average behavior is better fitted by including the temperature variation. On the other hand, figure 7(b) shows an orbit where inclusion of the temperature variation makes the fit worse. Figure $7(\mathrm{c})$ shows the difference over the whole orbit and indicates that there is little practical difference near perigee. The ambiguity of these results may be due to the placement of the thermistor on the outer housing of the antechamber-ion-source assembly. Although this gives a general indication of the temperature in the relevant region, it is probable that temperature 
differences of the order of $2^{\circ}$ or $3^{\circ}$ occur, and that the phase of temperature variation around the orbit at the thermistor might be somewhat different than that inside the antechamber itself.

There is a suggestive correlation between the period of the variation in the $a$ parameter and the variations in many spacecraft parameters as a result of the slow $\left(2^{\circ} /\right.$ day) drift of the orbit plane with respect to the Earth-Sun line. If one considers a given point in the orbit, say apogee, the angles between the spectrometer orifice and the Sun or solar panels and the measured source temperature all have cyclic variations similar to that of the $a$ parameter.

A possible cause of variation in density slope near apogee would be detection by the spectrometer of outgassing from the solar panels. Two arguments against this are the lack of detectable variation in the mass 28 peak at apogee during OPEP scans and the fact that the minimum value of parameter $a$ occurs when the orifice looks at the body before apogee and away from the body after apogee. The latter condition is always associated with noon-midnight orbits.

Figure 8 shows a typical orbit, comparing the observed mass 28 source density with model estimates of the contribution of $\mathrm{CO}$ to that source density. Figure 9 shows the fractional error in calculated molecular nitrogen ambient density due to errors in $a$ for the data in figure 8. Errors are compared with results from full surface analysis. Note that errors in $a$ affect calculated ambient density more severely after perigee and that the calculated ambient density is relatively insensitive to large excursions in $a$. This effect is the inverse of the sensitivity of $a$ to errors in the data.

It is clear from figure 4 that the $\mathrm{CO}$ model described is capable of giving a reasonable fit to the measured $\mathrm{CO}$ density over an orbit. The problem arises in later orbits when $\mathrm{CO}$ density near perigee becomes small, with large errors, and only the apogee density can be used in determining parameters

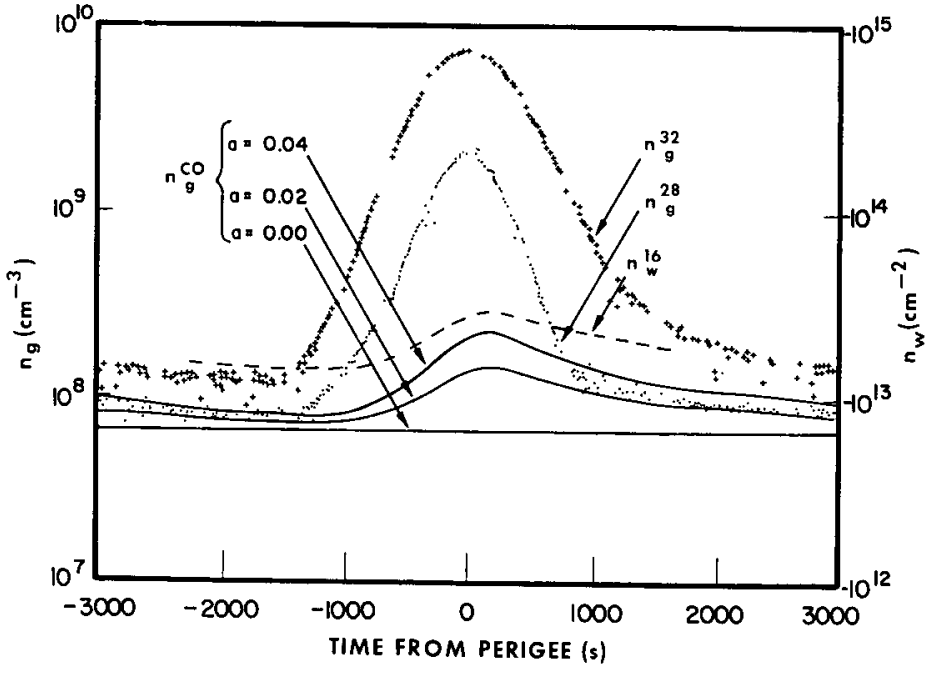

Figure 8.-Comparison of mass 28 source density with predicted $\mathrm{CO}$ density for several values of $a$. The calculated variation of atomic oxygen wall density and measured source densities for masses 32 and 28 are shown.

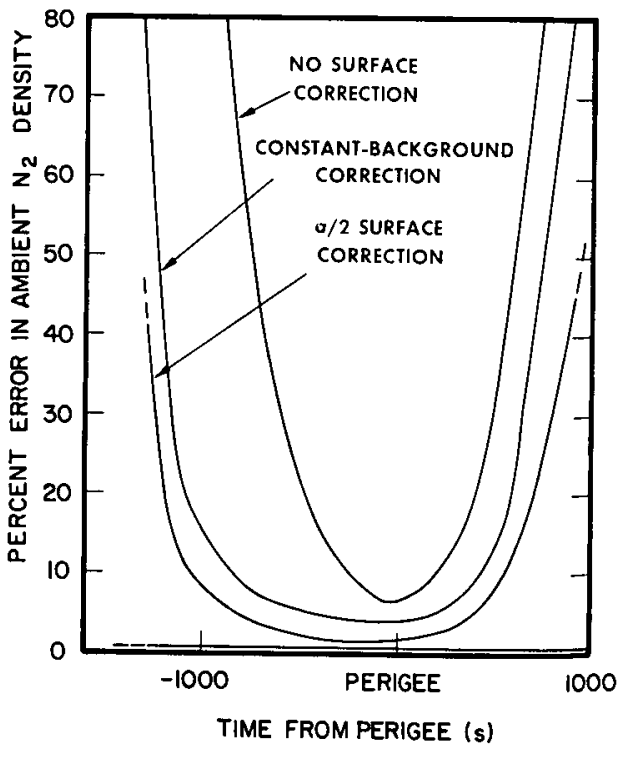

Figure 9.-Sensitivity of calculated $\mathrm{N}_{2}$ ambient density to changes in surface parameters. 
of the model. The small CO density at perigee means that the correction is likewise small at perigee, but the $\mathrm{CO}$ prediction becomes critical at higher altitudes and its accuracy is one of the factors that limits the altitude of reliable $\mathrm{N}_{2}$ densities. Because of the danger that variations in the $a$ parameter may be the result of extraneous effects not included in the model, a smooth curve was drawn through the values, as indicated in figure 6, and a 50-percent error bar was imposed on the CO correction (interpreted as the difference between the predicted CO and the apogee CO) after day 180 of 1969 . For the earlier orbits, the $\mathrm{CO}$ correction was taken to be accurate to 25 percent. For reliable estimates of $\mathrm{N}_{2}$ densities, it appears that a reasonable correction for $\mathrm{CO}$ can be made with the method described and that the use of $\mathrm{N}_{2}$ density estimates with large corrections can be avoided.

\section{ATOMIC OXYGEN DENSITY}

A model of the atomic oxygen interactions in the OGO 6 mass spectrometer has been constructed that accounts for the general behavior of the data. The model is a quasi-equilibrium model that ignores density gradients in the antechamber and variations in surface properties. It is applicable only after approximately 20 days of flight when reactions producing $\mathrm{CO}$ and $\mathrm{CO}_{2}$ become an insignificant loss process for oxygen. The model assumes that the flux of particles into the antechamber from the ambient atmosphere is primarily atomic oxygen with an insignificant component of molecular oxygen. The principal processes are expected to be adsorption of atomic oxygen and gas atom-adatom recombination, with a small amount of adatom-adatom recombination (Wood, Baker, and Wise, 1970). Desorption of atomic oxygen is included to explain the finite atomic oxygen source density at apogee. The in teraction of molecular oxygen with the surfaces is ignored, and recombined oxygen is assumed to appear immediately in the gas phase, because molecular oxygen is not expected to chemisorb on gold (Trapnell, 1953; Wood, Baker, and Wise, 1970) and would thus have a very short surface interaction time.

The rate of adsorption of atomic oxygen on the antechamber surfaces is proportional to the rate at which gas atoms strike the surface. From elementary kinetic theory, the collision frequency per unit area of a gas with volume number density $n_{g}$ and mean thermal speed $\bar{c}$ is $n_{g} \bar{c} / 4$. If $n$ represents the number of potential sites (usually taken to be $10^{15} \mathrm{~cm}^{-2}$ ) and $n_{w}$ the number of sites that are occupied, then the proportionality constant is $\left(1-n_{w} / n\right) k$, where $k$, the sticking coefficient, is regarded as constant (Langmuir isotherm). It was found that the oxygen surface coverage, using parameters that gave reasonable agreement with the data, was always of the order of $10^{14} \mathrm{~cm}^{-2}$ or less, and thus the factor $\left(1-n_{w} / n\right)$ was taken equal to 1 .

The rate of gas atom-adatom recombination is proportional to the collision rate of gas atoms with the wall and to the number of adsorbed atoms per unit area. The rate of formation of molecules is thus $\sigma_{a g} n_{g} n_{w} \bar{c} / 4$, where $\sigma_{a g}$ is the recombination coefficient. The rate of adatom-adatom recombination is proportional to the square of the wall coverage, and thus the rate of formation of molecules is given by $\sigma_{a a}\left(n_{w}\right)^{2}$, where $\sigma_{a a}$ is the recombination coefficient. The adatom-adatom recombination rate is a function of temperature such that $\sigma_{a a}$ is proportional to $\exp (-E / R T)$, where $E$ is the energy required for surface diffusion and reaction activation, $R$ is the gas constant, and $T$ is the absolute temperature. The desorption of surface atoms occurs at a rate given by $n_{w} / \tau$, where $\tau$ is a desorption time constant. The time constant is a function of temperature and is given approximately by $\tau=10^{-13} \exp (Q / R T)$, where $Q$ is the adsorption energy. 
Three equations can be written: one which relates the antechamber density of atomic oxygen to its sources and sinks, one relating molecular oxygen to its sources and sinks, and a similar equation for the wall density of atomic oxygen:

$$
\begin{aligned}
& V \frac{d n_{g}^{16}}{d t}=\frac{n_{a}^{16} \bar{c}_{a}^{16} A_{h}}{4}-\frac{n_{g}^{16} \bar{c}_{g}^{16} A_{h}}{4}+\frac{n_{w}^{16} A_{w}}{\tau_{w}^{16}}-\frac{\sigma_{a g}^{32} n_{w}^{16} A_{w} n_{g}^{16} \bar{c}_{g}^{16}}{4} \\
& V \frac{d n_{g}^{32}}{d t}=\frac{\sigma_{a g}^{32} n_{w}^{16} A_{w} n_{g}^{16} \bar{c}_{g}^{16}}{4}+\sigma_{a a}^{32}\left(n_{w}^{16}\right)^{2} A_{w}-\frac{n_{g}^{32} \bar{c}_{g}^{32} A_{h}}{4} \\
& \left.\frac{d n_{w}^{16}}{d t}=\frac{k_{16} n_{g}^{16} \bar{c}_{g}^{16}}{4}\left(1-\sigma_{a g}^{32} n_{w}^{16}\right) n_{g}^{16} \bar{c}_{g}^{16} A_{w}\right)-\frac{\sigma_{a g}^{32} n_{w}^{16} n_{g}^{16} \bar{c}_{g}^{16}}{4}-\frac{n_{w}^{16}}{\tau_{w}^{16}}-2 \sigma_{a a}^{32}\left(n_{w}^{16}\right)^{2}
\end{aligned}
$$

It is now convenient to define the time constants:

$$
\begin{gathered}
\tau_{g}^{16}=\frac{4 V}{\bar{c}_{g}^{16} A_{h}} \approx 0.016 \\
\tau_{g}^{32}=\frac{4 V}{\bar{c}_{g}^{32} A_{h}} \approx 0.023 \\
\tau_{a}^{16}=\frac{4 V}{\bar{c}_{a}^{16} A_{h}} \approx 0.008
\end{gathered}
$$

Assuming that $d n_{g}^{16} / d t$ is small with respect to $n_{g}^{16} / \tau_{g}^{16}$ and $d n_{g}^{32} / d t$ is small with respect to $n_{g}^{32} / \tau_{g}^{32}$, then equations (18), (19), and (20) can be rearranged and written as

$$
\begin{aligned}
\frac{n_{a}^{16}}{\tau_{a}^{16}} & =\frac{n_{g}^{16}}{\tau_{g}^{16}}-\frac{n_{w}^{16} A_{w}}{\tau_{w}^{16} V}+\frac{k_{16} A_{w} n_{g}^{16}}{\tau_{g}^{16} A_{h}}\left(1-\sigma_{a g}^{32} n_{w}^{16}\right)+\frac{\sigma_{a g}^{32} n_{w}^{16} n_{g}^{16} A_{w}}{\tau_{g}^{16} A_{h}} \\
\frac{n_{g}^{32}}{\tau_{g}^{32}} & =\frac{\sigma_{a g}^{32} n_{w}^{16} n_{g}^{16} A_{w}}{\tau_{g}^{16} A_{h}}+\frac{A_{w} \sigma_{a a}^{32}\left(n_{w}^{16}\right)^{2}}{V} \\
\frac{d n_{w}^{16}}{d t} & =\frac{k_{16} V n_{g}^{16}}{\tau_{g}^{16} A_{h}}\left(1-\sigma_{a g}^{32} n_{w}^{16}\right)-\frac{n_{w}^{16}}{\tau_{w}^{16}}-\frac{\sigma_{a g}^{32} n_{w}^{16} n_{g}^{16} V}{\tau_{g}^{16} A_{h}}-2 \sigma_{a a}^{32}\left(n_{w}^{16}\right)^{2}
\end{aligned}
$$


Now, if $\left(-A_{h} / V\right)$ times equation (23) is added to equation (21) and if twice equation (22) is added to the previous sum, the following general result can be obtained:

$$
\frac{n_{a}^{16}}{\tau_{a}^{16}}=\frac{n_{g}^{16}}{\tau_{g}^{16}}+2 \frac{n_{g}^{32}}{\tau_{g}^{32}}+\frac{A_{w}}{V} \frac{d n_{w}^{16}}{d t}
$$

The ambient atomic oxygen is the sum of a density derived from the source density of atomic oxygen, twice the source density of molecular oxygen, and a correction proportional to the rate of change of the oxygen wall density.

Determination of the wall coverage is found in principle by integrating equation (23), given the four surface parameters $\left(k_{16}, \sigma_{a g}^{32}, \sigma_{a a}^{32}\right.$, and $\left.\tau_{w}^{16}\right)$ and values of $n_{g}^{16}$. There are three possible sources for $n_{g}^{16}$ values: (1) the mass 16 source density measurements (subtracting the portion due to the fractionation of molecular oxygen), (2) equation (22) (using mass 32 source density measurements $\left(n_{g}^{32}\right)$, and (3) equation (21) (using an atmospheric model for $n_{a}^{16}$ ). The first possibility was avoided, because $n_{g}^{16}$ would then be the difference of large numbers and because the fractionation ratio was not known with complete certainty. The second possibility was initially considered the best, because it uses measurements having the least scatter. The third possibility suffers the disadvantage of requiring knowledge of $n_{a}^{16}$, the quantity one is attempting to measure.

An examination of the various equations shows that the surface parameters should, in principle, be determinable from the data. If it is assumed that adatom-gas atom recombination dominates over adatom-adatom recombination near perigee (as found by Wood, Baker, and Wise, 1970), derivation from equation (22) gives

$$
\sigma_{a g}^{32} n_{w}^{16}=\frac{A_{h} \tau_{g}^{16} n_{g}^{32}}{A_{w} \tau_{g}^{32} n_{g}^{16}}
$$

and this is roughly 0.022 . Also, near perigee one finds from equation (23) that $k_{16}$ is roughly equal to $\sigma_{a g}^{32} n_{w}^{16}$ when $\sigma_{a a}^{32}$ and $1 / \tau_{w}^{16}$ are small.

The apogee data place further constraints on the parameters. The existence of a finite $n_{g}^{16}$ implies a finite $\tau_{w}^{16}$, which from equation (21) is approximately

$$
\tau_{w}^{16}=\frac{\tau_{g}^{16} A_{h} n_{w}^{16}}{2 V \sigma_{a g}^{32} n_{w}^{16} n_{g}^{16}}
$$

assuming $n_{a}^{16}$ is zero. Assuming also that the apogee mass 32 density is primarily due to adatomadatom recombination, one can derive from equations (22) and (24) the values of $n_{w}^{16}$ at apogee, and $\sigma_{a a}^{32}:$

$$
\begin{aligned}
& n_{w}^{16}=-\frac{4 V\left(\dot{n}_{g}^{32}\right)^{2}}{\tau_{g}^{32} A_{w} d n_{g}^{32} / d t} \\
& \sigma_{a a}^{32}=\frac{V n_{g}^{32}}{A_{w} \tau_{g}^{32}\left(n_{w}^{16}\right)^{2}}
\end{aligned}
$$




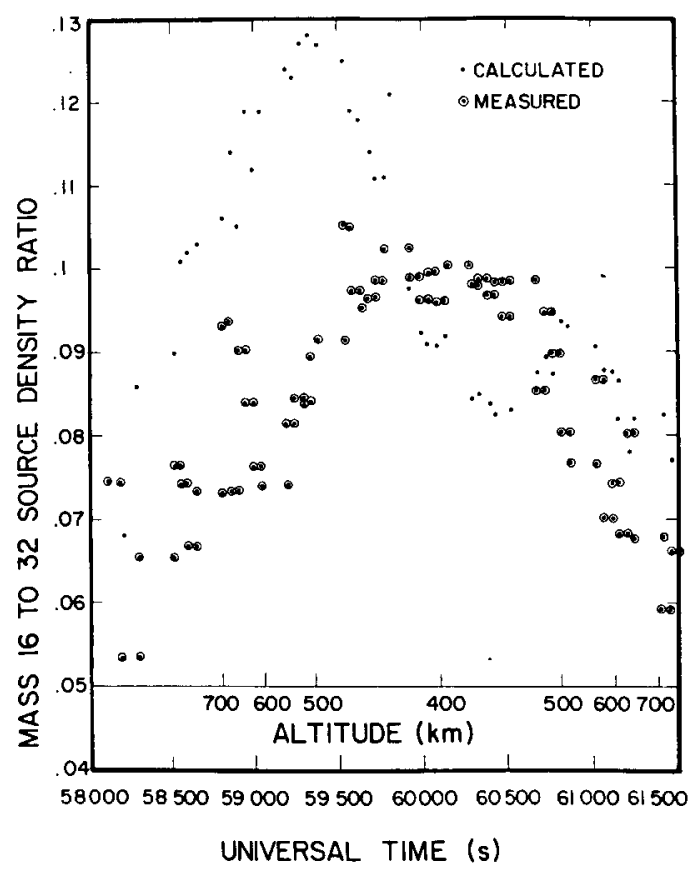

Figure 10.-Measured and calculated mass 16 to 32 source-density ratios.

If it is further assumed that perigee and apogee wall densities are the same order of magnitude, rough values of all the surface parameters will have been determined.

An attempt was made to refine these approximate values of the surface parameters by a least squares fit of all the mass 16 source densities in an orbit and the mass 32 source densities at apogee. Values of $n_{g}^{16}$ were predicted from equation (23), using measured $n_{g}^{32}$ and values of $n_{w}^{16}$ calculated from equation (23). Values of $n_{g}^{32}$ near apogee were predicted by determining $n_{g}^{16}$ from equation (21) with $n_{a}^{16}=0$ and substituting it into equation (22). Equation (23) was solved for $n_{w}^{16}$ by repeated numerical integration, using values of $n_{g}^{16}$ calculated from equation (22), until the $n_{w}^{16}$ values were the same at the beginning and end of an orbit. Whereas parameters could be found that improved the fit to the data over the rough parameters estimated above, it became apparent that a systematic variation in the residuals (differences between measured and predicted values) was frustrating the search for a satisfactory solution.

The nature of the problem is best seen by examining the ratios of mass 16 to 32 source densities. A plot of these ratios versus time for a whole orbit is shown in figure 10, which is characteristic of all orbits after the first month. The observed ratios are remarkably symmetric about perigee and have a maximum at perigee. The predicted values of the ratio shown are not the best that can be achieved with a least squares fit, but serve to illustrate the problem. The predicted ratio has a slight dip after perigee and is higher before perigee than after, contrary to the near-perigee behavior of the measured ratio. The reason for the near-perigee behavior of the predicted ratio can be seen from equation (25). Assuming that molecular oxygen is the result only of adatom-gas atom recombination (independent of how the $n_{g}^{16}$ level is established), the $n_{g}^{16} / n_{g}^{32}$ ratio is inversely proportional to the wall density $n_{w}^{16}$. Because the sources contributing to the wall density increase to a maximum near perigee, the wall 
density should likewise increase toward and beyond perigee to reach a maximum where the sources and sinks are equal. This behavior is reflected in the predicted values of figure 10 . A limiting case would exist if the wall density were constant, implying constant $n_{g}^{16} / n_{g}^{32}$. In an attempt to explain the behavior of the oxygen ratio, a number of speculative processes were considered, including direct surface ionization of wall oxygen in the source, dissociative adsorption of $\mathrm{O}_{2}$, and nonhomogeneous distribution of ambient flux on the antechamber walls; but none was satisfactory. A process that would provide maximum $n_{g}^{16} / n_{g}^{32}$ at perigee would involve the reaction of oxygen atoms (surface or gas phase) with some other species that peaks at perigee more strongly than atomic oxygen, resulting in a molecule that carries atomic oxygen into the ion source, where it is dissociatively ionized. Neither of these possibilities could be established from the data with certainty or used in a quantitative manner, and attempts to fit the data with a method that relied largely on the mass 16 to 32 source-density ratio were abandoned.

The method finally used included fitting only the mass 32 source densities over the whole orbit, calculating $n_{g}^{32}$ from equation (22), using $n_{g}^{16}$ from equation (21) as

$$
\frac{n_{g}^{16}}{\tau_{g}^{16}}=\frac{n_{a}^{16} / \tau_{a}^{16}+n_{w}^{16} A_{w} / \tau_{w}^{16} V}{1+k_{16}\left(1-\sigma_{a g}^{32} n_{w}^{16}\right) A_{w} / A_{h}+\sigma_{a g}^{32} n_{w}^{16} A_{w} / A_{h}}
$$

and obtaining $n_{w}^{16}$ from equation (23) as before. Lacking the measured mass 16 source density, the surface parameters cannot be determined without using a model atmosphere for $n_{a}^{16}$. Fortunately, it can be shown that independent knowledge of all the surface parameters is not necessary to determine the density correction. Only the ratio of $k_{16}$ to $\sigma_{a g}^{32}$ is important in the correction formula if $k_{16}$ and $\sigma_{a g}^{32} n_{w}^{16}$ are small with respect to 1 . If equation (22) is solved for $n_{g}^{16}$ and substituted in equation (23), the important terms will depend only on the $k_{16} / \sigma_{a g}^{32}$ ratio. Similarly, from equation (29), with $k_{16} A_{w} / A_{h}$ large with respect to 1 , the substitution into equation (23) again produces ratios of $k_{16}$ to $\sigma_{a g}^{32}$. Thus it was decided to fix $k_{16}$ at 0.022 , which gives rough agreement with the perigee $n_{g}^{16} / n_{g}^{32}$ ratio. The value determined by Wood, Baker, and Wise (1970) was 0.044 .

It is also very difficult to separate the two wall-loss terms $n_{w}^{16} / \tau_{w}^{16}$ and $2 \sigma_{a a}^{32}\left(n_{w}^{16} \cdot\right)^{2}$ because at apogee, where they are important, the wall density is not changing fast enough to provide a significant difference between linear and square-law loss. It was decided to fix $1 / \tau_{w}^{16}$ at $6 \times 10^{-5}$, which provides an approximate agreement with the apogee mass 16 source density. Numerical experiments showed that within wide ranges the chosen values of $k_{16}$ and $\tau_{w}^{16}$ made no practical difference to the oxygen correction as long as the remaining two parameters were adjusted for best fit to the mass 32 data. Only two surface parameters are still to be determined: $\sigma_{a g}^{32}$ and $\sigma_{a a}^{32}$.

The model chosen to provide the ambient atomic oxygen densities was basically the static diffusion model of Jacchia (1965), and orbits were selected that, as far as possible, had $\mathrm{N}_{2}$ densities in reasonable agreement with the model. This was accomplished by comparing the exospheric temperature, which yielded the measured $\mathrm{N}_{2}$ density, with the exospheric temperature as predicted by Jacchia. The temperature used for predicting $\mathrm{N}_{2}$ and atomic oxygen could then be corrected to provide better agreement with the measured $\mathrm{N}_{2}$. An attempt was made, also, to pick orbits that had perigees near the Equator and low magnetic activity. Five orbits were selected for the determination of $\sigma_{a g}^{32}$ and $\sigma_{a a}^{32}$. These occurred on Sept. 22, 24, 26, and Nov. 15, 1969, and on Mar. 22, 1970. The difference between the temperature needed to derive the measured nitrogen density $T_{M}$ and the model temperature $T_{J}$ is 


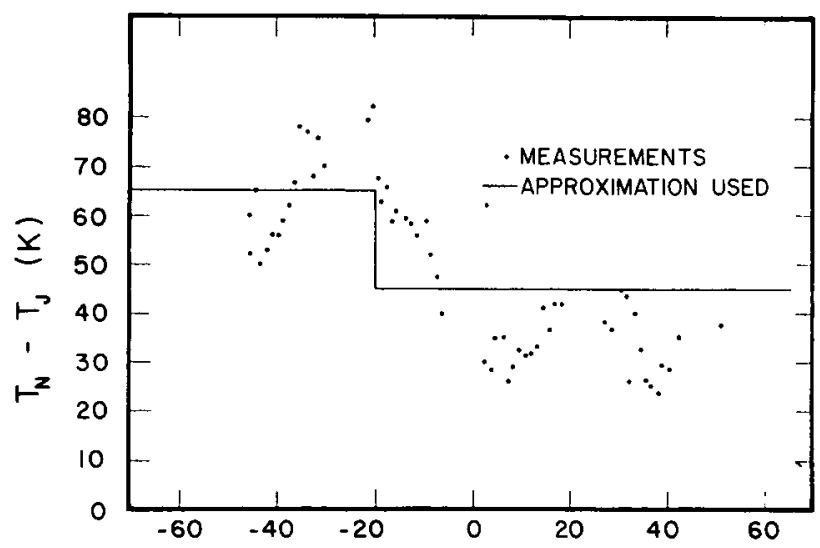

(a)

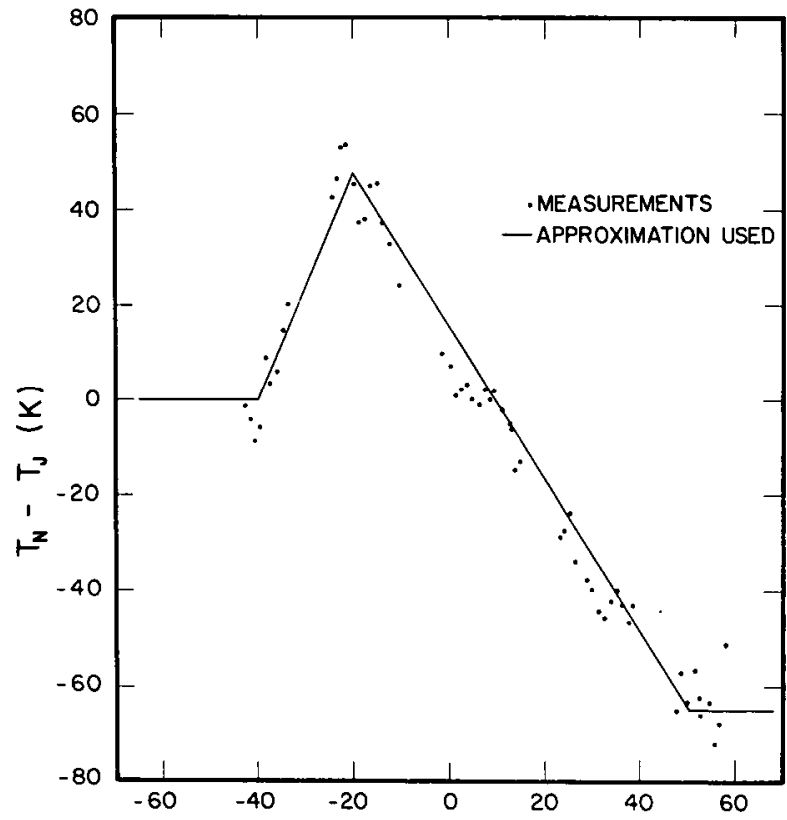

(c)

LATITUDE (deg)

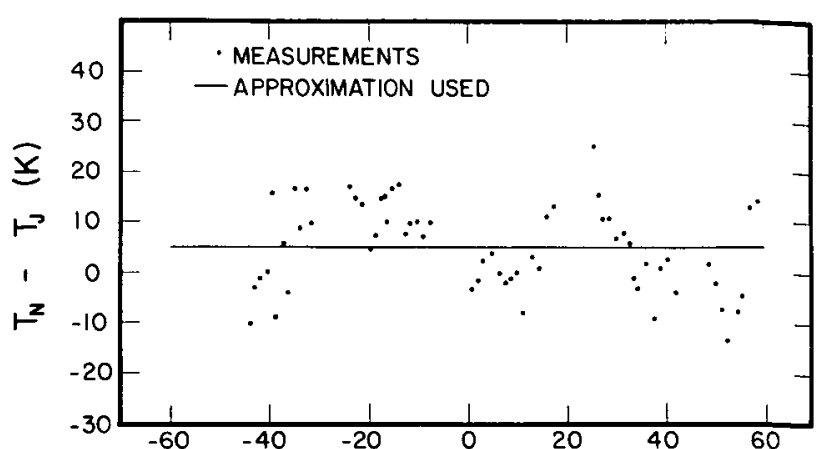

(b)

LATITUDE (deg)

Figure 11.-Comparison of nitrogen ambient density with Jacchia model. (a) Orbit of Sept. 22, 1969. (b) Orbit of Sept. 24, 1969. (c) Orbit of Sept. 26, 1969.

plotted against latitude in figure 11. The approximations used for the model temperature when predicting atomic oxygen densities are also shown. The predicted atomic oxygen densities were scaled with a multiplicative factor so that only the predicted oxygen latitudinal distribution and not absolute values would be used in determining surface parameters. This factor and the unknown surface parameters were determined by a least squares fit to measured source densities for the selected orbits.

Values of oxygen surface parameters $\sigma_{a g}^{32}$ and $\sigma_{a a}^{32}$ determined for the five selected orbits are given in table 1. Two surface parameters determined from the orbit on Sept. 24 were chosen as the values to be used in ambient density processing because the $\mathrm{N}_{2}$ densities agreed most closely with the model predictions, the orbit was during equinox when cross-Equator gradients are least likely, and the orbital 
Table 1.-Surface Parameters for Oxygen ${ }^{a}$

\begin{tabular}{|c|c|c|}
\hline Date & \multicolumn{1}{|c|}{$\sigma_{a a}^{32}$} & $\sigma_{a g}^{32}$ \\
\hline Sept. 22, 1969 & $8.67 \times 10^{-19}$ & $3.72 \times 10^{-16}$ \\
Sept. 24, 1969 & $12.00 \times 10^{-19}$ & $3.81 \times 10^{-16}$ \\
Sept. 26, 1969 & $4.52 \times 10^{-19}$ & $2.80 \times 10^{-16}$ \\
Nov. 15, 1969 & $2.20 \times 10^{-19}$ & $1.99 \times 10^{-16}$ \\
Mar. 22, 1970 & $20.60 \times 10^{-19}$ & $4.63 \times 10^{-16}$ \\
\hline
\end{tabular}

${ }^{\mathrm{a}}$ Based on assumed values of $k_{16}=0.022$ and $1 / \tau_{w}^{16}=6 \times 10^{-5}$.

variation of densities indicated that this was an unusually quiet time period (in agreement with the magnetic activity indices). The comparison between the measured and predicted source densities for the Sept. 24 orbit is shown in figure 12 ; these correspond to mass 16 to 32 source-density ratios plotted versus time in figure 10 . The predicted variation in wall density $n^{16}$ for this orbit is given in figure 13.

Rather than calculate ambient densities directly from equation (29), it was decided to assume that $n_{a}^{16}$ was always zero at apogee and to subtract the results of equation (24) for the apogee case (subscript ap) from the results for other times:

$$
\frac{n_{a}^{16}}{\tau_{a}^{16}}=\frac{n_{g}^{16}-\left(n_{g}^{16}\right)_{\mathrm{ap}}}{\tau_{g}^{16}}+2 \frac{n_{g}^{32}-\left(n_{g}^{32}\right)_{\mathrm{ap}}}{\tau_{g}^{32}}+\frac{A_{w}}{V}\left[\frac{d n_{w}^{16}}{d t}-\left(\frac{d n_{w}^{16}}{d t}\right)_{\mathrm{ap}}\right]
$$

This procedure has the virtue of insuring that the ambient density tends to zero at apogee regardless of small errors in calculating $d n_{w}^{16} / d t$. Perigee results will be essentially unchanged. A correction factor was then defined as follows:

$$
\mathrm{CF}=\frac{\left(A_{w} / V\right)\left[d n_{w}^{16} / d t-\left(d n_{w}^{16} / d t\right)_{\mathrm{ap}}\right]}{\left[n_{g}^{16}-\left(n_{g}^{16}\right)_{\mathrm{ap}}\right] / \tau_{g}^{16}+2\left[n_{g}^{32}-\left(n_{g}^{32}\right)_{\mathrm{ap}}\right] / \tau^{32}}
$$

which means that if ambient densities are calculated by the usual method of ignoring surface effects but subtracting the background (the first two terms of eq. (30)), the densities corrected for surface effects will be $(1+C F)$ times the density determined from the first two terms of equation (30). A plot of CF versus time is shown in figure 14 .

The effect of an arbitrary change in surface parameters is demonstrated in figure 15 by examining the ratio of corrected ambient density to the model ambient density. Note the insensitivity of measured density near $500 \mathrm{~km}$ on the outgoing portion of the orbit to changes in surface parameters, which is in agreement with the correction being very low here.

The ambient densities derived for other orbits using the Sept. 24 surface parameters are compared in figure 16 with the densities derived using the best-fit parameters obtained from that particular orbit. There is no large discrepancy except for the Nov. 15 orbit, which, being nearer to solstice than to equinox, may have composition effects not accounted for in the static diffusion models. The 


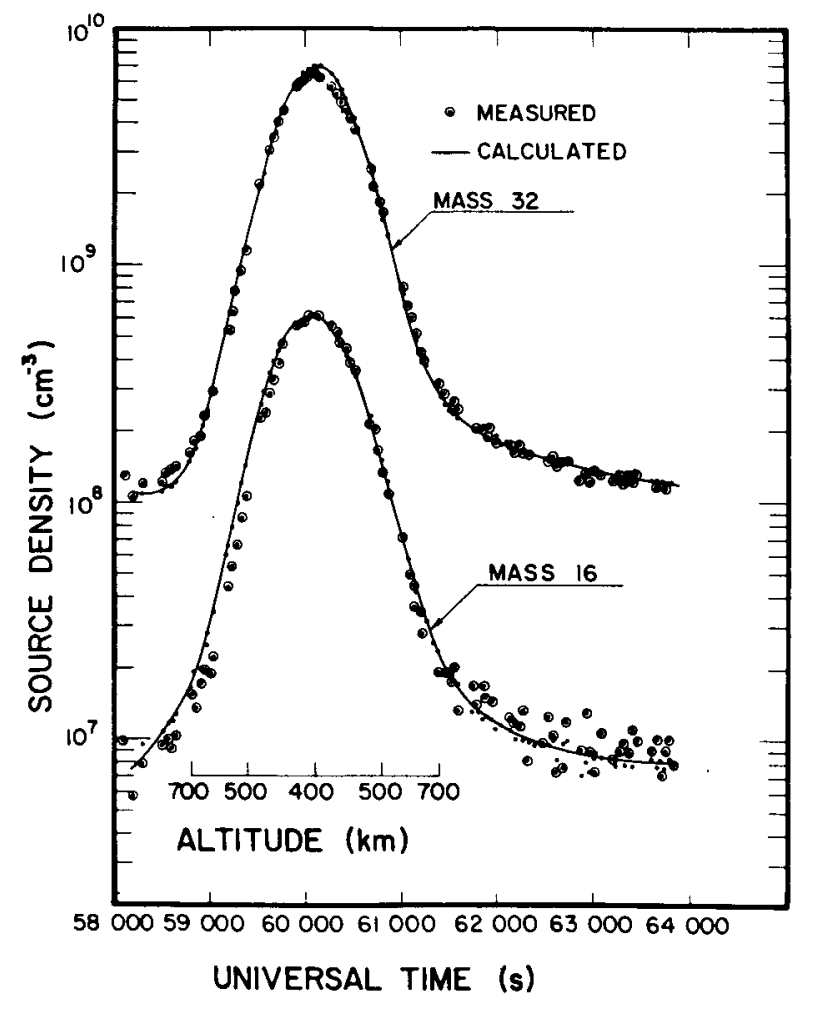

Figure 12.-Comparison of measured source density with densities from least squares fit to equations (22) and (24).

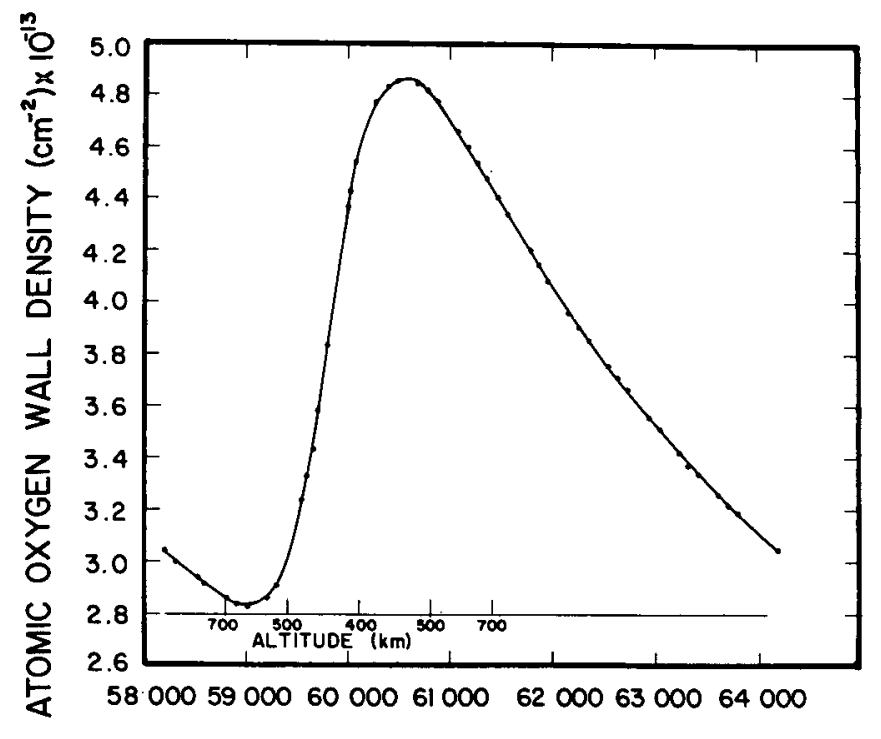

UNIVERSAL TIME (s)

Figure 13.-Calculated atomic oxygen wall density for the orbit of figure 12. 


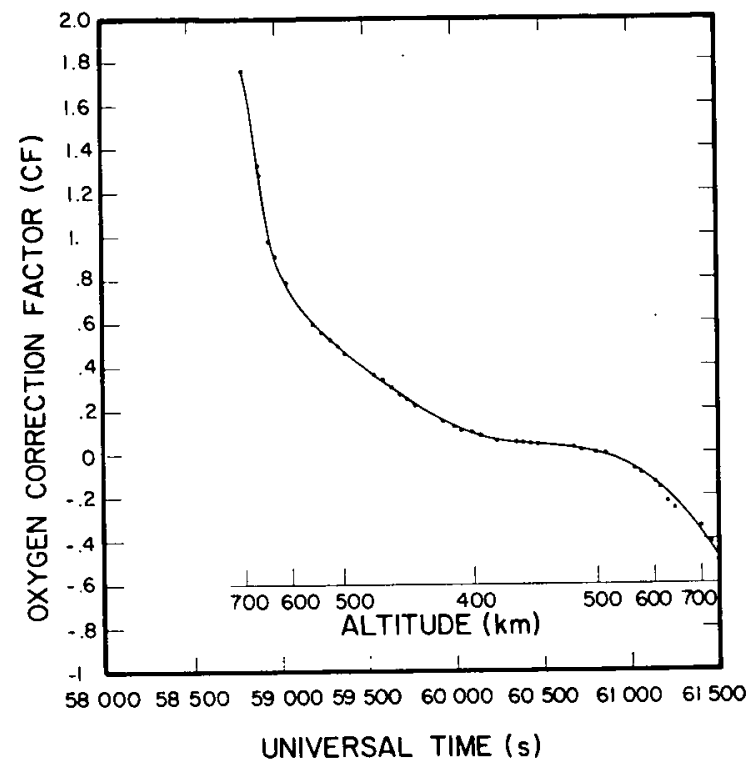

Figure 14.-Oxygen correction factor (CF) for the orbit of figure 12.

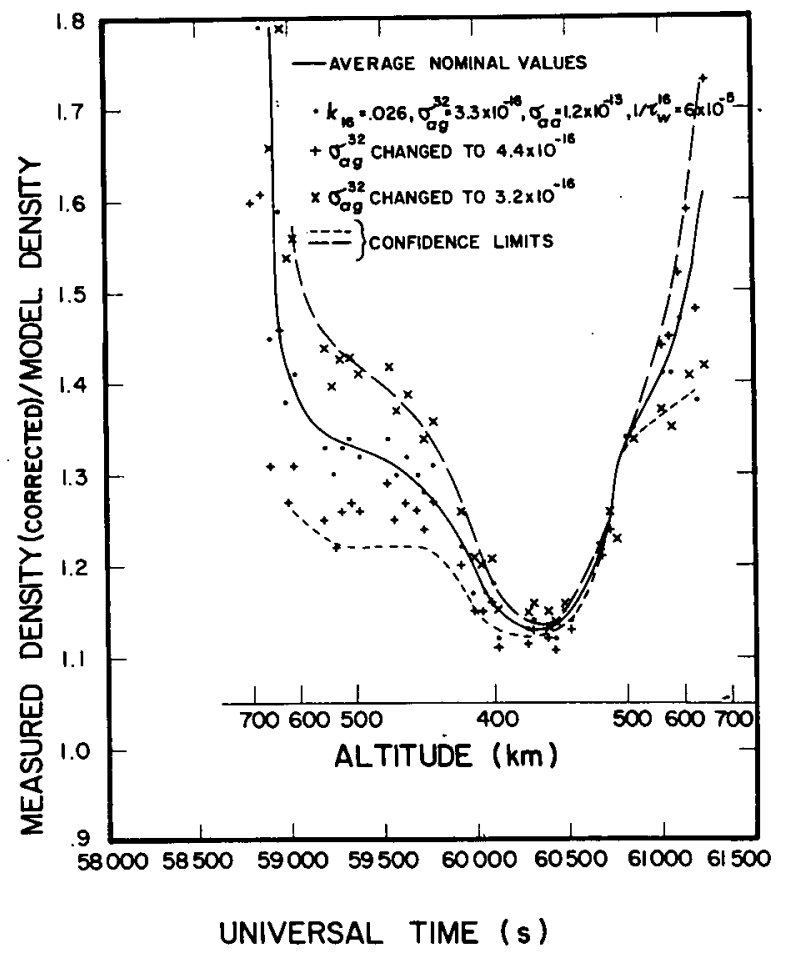

Figure 15.-Effect of surface parameter changes on calculated ambient density of atomic oxygen. 


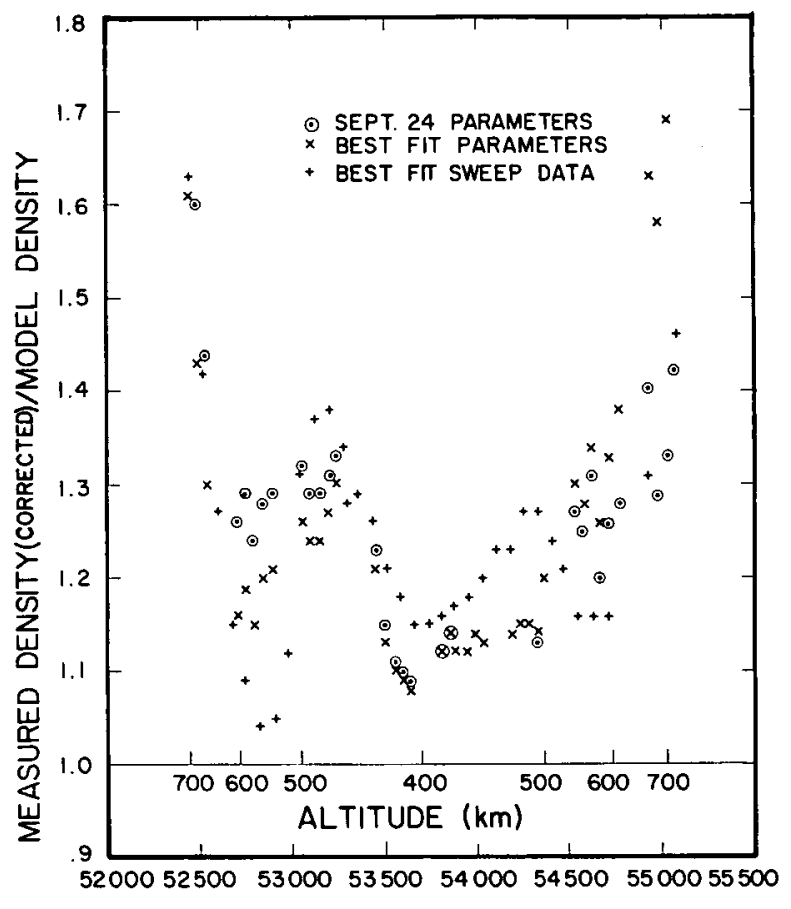

(a)

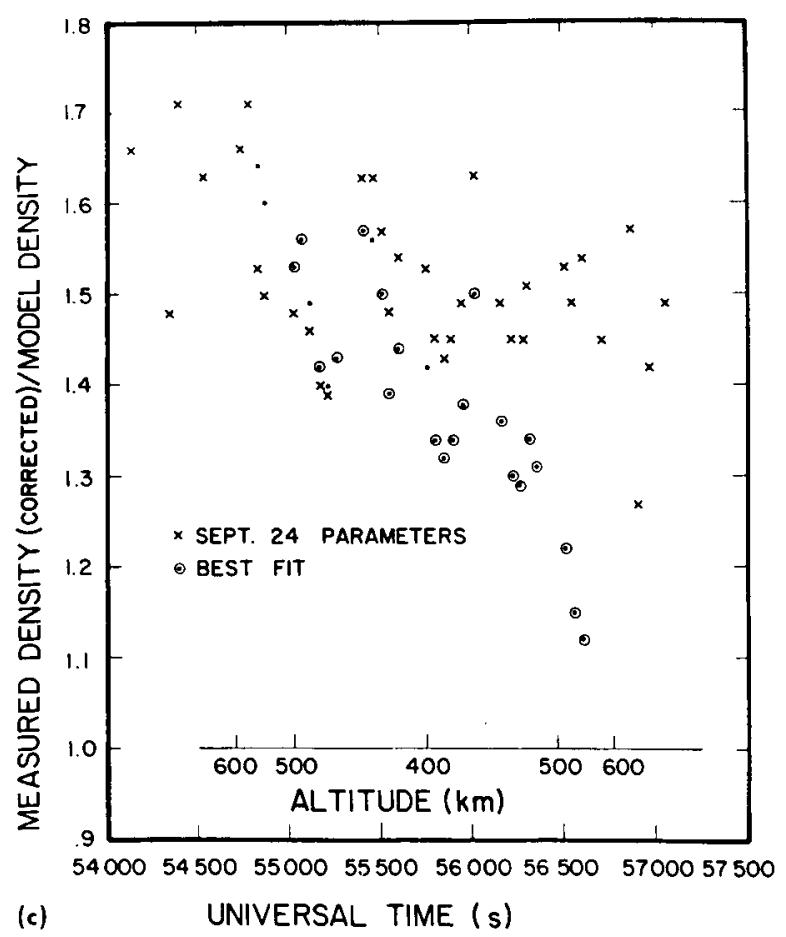

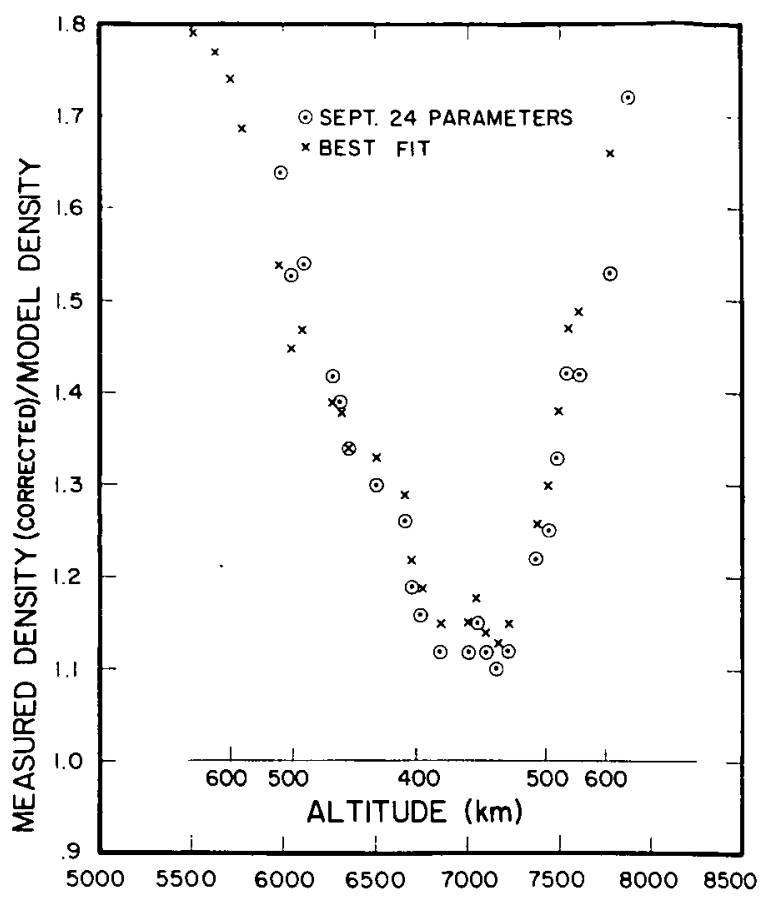

(b)

UNIVERSAL TIME (s)

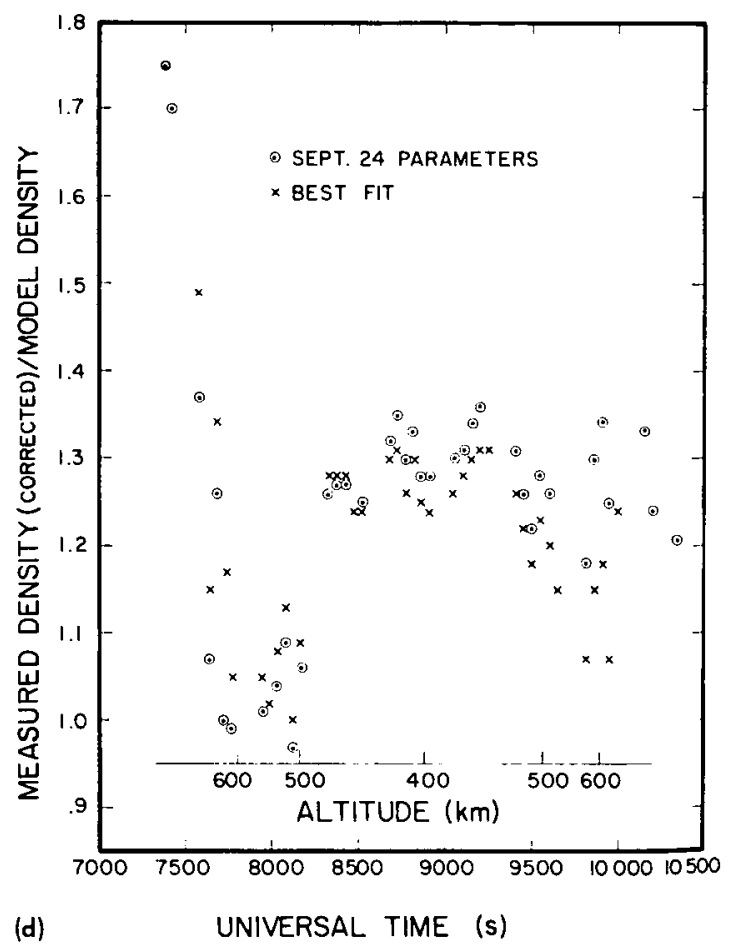

Figure 16.-Comparison of ambient densities derived from Sept. 24 surface parameters and from best fit surface parameters from orbits on other dates. (a) Best fit data from Sept. 22 orbit and sweep data. (b) Best fit data from selected orbit on Sept. 26. (c) Best fit data from selected orbit on Nov. 15. (d) Best fit data from selected orbit on Mar. 22, 1970. 
comparison for the Mar. 22 equinox orbit is quite satisfactory. The fact that different pairs of values for $\sigma_{a g}^{32}$ and $\sigma_{a a}^{32}$ can give substantially the same ambient densities suggests that there is an effective correlation between the two parameters. This is illustrated in figure 17, where the line represents sets of parameters for Sept. 24 derived by arbitrarily changing one parameter and least square fitting for the remaining one. The parameters for the other days fall very close to this line. Thus, with the method used, the surface model has in effect only one degree of freedom.

The extent to which the corrected ambient densities (determined from the measurements) depend on the model ambient densities used in determining the surface parameters was studied by making arbitrary changes in the model temperature used to predict the model density. It was found that adding or subtracting a constant temperature to the Jacchia model or using a constant temperature throughout the orbit made no significant difference in the final corrected ambient densities, although the fit to the source density was not always very good, and different surface parameters resulted. However, if a temperature gradient of $\pm 1 \mathrm{~K}$ per degree latitude was added to Jacchia-model temperatures, it was found that this gradient was reflected in the corrected density measurements. The same happened for $\pm 2 \mathrm{~K}$, but the degree of fit to the source densities was sufficiently poor to suggest that a gradient of this magnitude was unlikely in the real atmosphere for the orbit tested. The result of these tests is that an error in the ambient density model, which is symmetric about perigee, is unable to cause a significant error in the corrected measured density, but an error in the form of a temperature gradient would be reflected in the measured density.

An assessment of the error resulting from the electronic failure of Aug. 13, 1969, was made by hand-constructing a full-orbit curve of mass 32 source density from the sweep mass 32 source density, as opposed to using that obtained in the step mode, and by using these data to determine ambient density. Presumably the sweep determinations would be more accurate at the higher altitudes. The densities beyond $600 \mathrm{~km}$ using the sweep data were systematically lower than those from the step data. On Sept. 22 the differences were roughly 10 percent at $600 \mathrm{~km}$ and 20 percent at $700 \mathrm{~km}$ (fig. 16(a)). On Sept. 26, the corresponding differences were 15 and 35 percent. In addition, the difference between before and after perigee densities at $600 \mathrm{~km}$ was about 10 percent smaller using the sweep data.

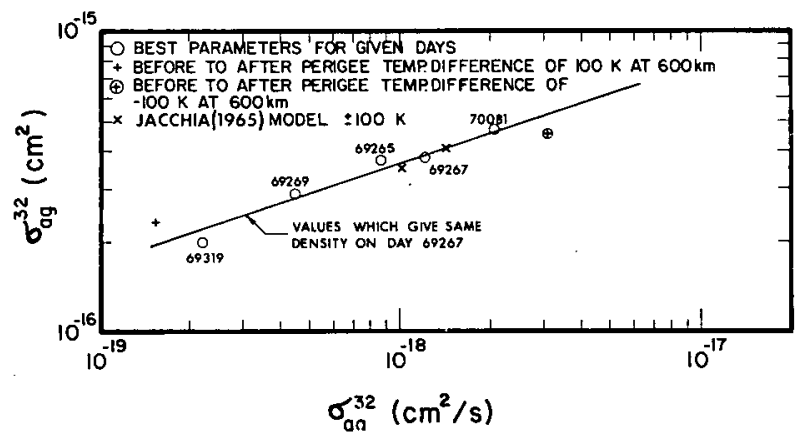

Figure 17.-Comparison of $\sigma_{a g}^{32}$ and $\sigma_{a a}^{32}$, assuming

$$
k_{16}=0.022 \text { and } 1 / \tau_{w}^{16}=6 \times 10^{-5} \text {. }
$$


During certain time periods (e.g., Nov. 1969) there were changes near apogee in the slope of the source density versus time plots which suggested that some process in addition to simple desorption was occurring. Maximum deviations in density were on the order of 15 percent. Three possible explanations were considered: (1) temperature variation of desorption and adatom-adatom recombination, (2) outgassing from the body or solar panels of the spacecraft, and (3) solar radiation entering the spectrometer antechamber. Unfortunately, all three possible effects are highly correlated in practice, because the normal spacecraft operation was such that when the orifice was facing the Sun, the orifice was always facing in the direction of the solar paddles and main body. The phase was such that all three effects could qualitatively predict the perturbations in Nov. 1969, but would not be correct at other times. The ability to predict the temperature effects depends on knowledge of the surface interaction energies, and when these are estimated from the surface parameters already determined, the variations in source density caused by temperature appear to be too large. Ambient densities determined by including temperature variations differed from those determined without temperature variations by less than 10 percent up to $600 \mathrm{~km}$. There was some evidence for very small variations in source density during OPEP scans, which may suggest outgassing from the solar panels or body, but the data are too meager to give quantitative predictions for any time during an orbit.

In summary, a set of surface parameters has been determined that provides a reasonable fit to the mass 16 and 32 source densities and is consistent with the ambient atomic oxygen predicted by the static diffusion model (Jacchia, 1965) during equinox. It was found that parameters determined from orbits up to 6 months apart were reasonably consistent, which indicates, in conjunction with the constant mass 16 to 32 source-density ratio at perigee, that surface conditions are relatively stable after the first few weeks of flight. The surface model is not applicable to the first few weeks of flight because there was significant production of $\mathrm{CO}_{2}$ and $\mathrm{CO}$ that has not been taken into account.

Unfortunately the surface parameters could not be determined entirely from the source-density data, but depended to some degree on an assumed ambient density model. However, the gradient across perigee was the essential feature derived from the ambient density model, and it is probable that by using an orbit near equinox with perigee near the Equator the possible errors were minimized.

During routine processing, equation (23) is solved for $n_{w}^{16}$ by integrating numerically in serial fashion from orbit to orbit, without the need to repeat the process as was done for a single orbit, and the correction factor of equation (31) is calculated from equation (24) by use of the instantaneous $n_{w}^{16}$. To avoid using ambient densities with large corrections, it was decided to add to the density error a term equal to 25 percent of the correction, if positive, and 50 percent, if negative. The different error values arose because it appeared that a change in a single parameter had twice the effect on the correction when the correction was negative. 


\section{REFERENCES}

Carignan, G. R.; and Pinkus, W. H.: OGO-F-04 Experiment Description. Tech. Note 08041-3-T, Space Physics Res. Lab., Univ. of Michigan, Oct. 1968.

Ehrlich, Gert: "Molecular Dissociation and Reconstitution on Solids." J. Chem. Phys. 31: 111, 1959. Jacchia, L. G.: "Static Diffusion Models of the Upper Atmosphere With Empirical Temperature Profiles." Smithson. Contrib. Astrophys. 8: 215, 1965.

Kittel, Charles: Introduction to Solid State Physics. John Wiley \& Sons, Inc., 1968.

Moe, Kenneth; and Moe, Mildred M.: “The Effect of Adsorption on Densities Measured by Orbiting Pressure Gauges." Planet. Space Sci. 15: 1329, 1967.

Moe, Mildred M.; and Moe, Kenneth: "The Roles of Kinetic Theory and Gas-Surface Interactions in Measurements of Upper-Atmospheric Density." Planet. Space Sci. 17: 917, 1969.

Silverman, Peter J.; and Newton, George P.: "Relation of Measured Outgassing Pressures to Surface Adsorption in Satellite Borne Pressure Gauges." J. Vac. Sci. Technol. 7: 323, 1970.

Trapnell, B. M. W.: "The Activities of Evaporated Metal Films in Gas Chemisorption." Proc. Roy. Soc., Ser. A 218: 566, 1953.

Wood, Bernard J.; Baker, Bill R.; and Wise, Henry: Research Related to Measurements of Atomic Species in the Earth's Upper Atmosphere. Final Rept. (Contract NASr-49(30)), Stanford Res. Inst., 1970.

Zahn, U. von: "Mass Spectrometric Measurements of Atomic Oxygen in the Upper Atmosphere: A Critical Review." J: Geophys. Res. 72: 5933, 1967. 